\title{
G-protein-coupled estrogen receptor activation upregulates interleukin-1 receptor antagonist in the hippocampus after global cerebral ischemia: implications for neuronal self-defense
}

\author{
Ning Bai ${ }^{1}$, Quanguang Zhang ${ }^{2}$, Wenli Zhang ${ }^{1}$, Bin Liu ${ }^{3}$, Fang Yang ${ }^{1}$, Darrell Brann ${ }^{2^{*}}$ and Ruimin Wang ${ }^{1,4^{*}}$
}

\begin{abstract}
Background: G-protein-coupled estrogen receptor (GPER/GPR30) is a novel membrane-associated estrogen receptor that can induce rapid kinase signaling in various cells. Activation of GPER can prevent hippocampal neuronal cell death following transient global cerebral ischemia $(\mathrm{GCl})$, although the mechanisms remain unclear. In the current study, we sought to address whether GPER activation exerts potent anti-inflammatory effects in the rat hippocampus after $\mathrm{GCl}$ as a potential mechanism to limit neuronal cell death.

Methods: GCl was induced by four-vessel occlusion in ovariectomized female SD rats. Specific agonist G1 or antagonist G36 of GPER was administrated using minipump, and antisense oligonucleotide (AS) of interleukin-1 $\beta$ receptor antagonist (IL1RA) was administrated using brain infusion kit. Protein expression of IL1RA, NF-KB-P65, phosphorylation of CREB (p-CREB), $\mathrm{Bc} 12$, cleaved caspase 3, and microglial markers Iba1, CD11b, as well as inflammasome components NLRP3, ASC, cleaved caspase 1, and Cle-IL1 $\beta$ in the hippocampal CA1 region were investigated by immunofluorescent staining and Western blot analysis. The Duolink II in situ proximity ligation assay (PLA) was performed to detect the interaction between NLRP3 and ASC. Immunofluorescent staining for NeuN and TUNEL analysis were used to analyze neuronal survival and apoptosis, respectively. We performed Barnes maze and Novel object tests to compare the cognitive function of the rats.
\end{abstract}

Results: The results showed that G1 attenuated GCl-induced elevation of Iba1 and CD11b in the hippocampal CA1 region at 14 days of reperfusion, and this effect was blocked by G36. G1 treatment also markedly decreased expression of the NLRP3-ASC-caspase 1 inflammasome and IL1 $\beta$ activation, as well as downstream NF-KB signaling, the effects reversed by G36 administration. Intriguingly, G1 caused a robust elevation in neurons of a well-known endogenous anti-inflammatory factor IL1RA, which was reversed by G36 treatment. G1 also enhanced p-CREB level in the hippocampus, a transcription factor known to enhance expression of IL1RA. Finally, in vivo ILIRA-AS abolished the anti-inflammatory, neuroprotective, and anti-apoptotic effects of $\mathrm{G} 1$ after $\mathrm{GCl}$ and reversed the cognitive-enhancing effects of $\mathrm{G} 1$ at 14 days after $\mathrm{GCl}$.

Conclusions: Taken together, the current results suggest that GPER preserves cognitive function following GCI in part by exerting anti-inflammatory effects and enhancing the defense mechanism of neurons by upregulating IL1RA.

Keywords: Global cerebral ischemia, G-protein-coupled estrogen receptor 1 (GPER/GPR30), Inflammasome, NACHT-, LRR- and PYD-containing protein 3 (NLRP3), Interleukin-1 receptor antagonist (IL1RA)

\footnotetext{
* Correspondence: dbrann@augusta.edu; ruimin-wang@163.com

${ }^{2}$ Department of Neuroscience and Regenerative Medicine, Medical College

of Georgia, Augusta University, Augusta, GA 30912, USA

${ }^{1}$ Neurobiology Institute of Medical Research Center, North China University

of Science and Technology, Tangshan 063210, Hebei, China

Full list of author information is available at the end of the article
}

(c) The Author(s). 2020 Open Access This article is distributed under the terms of the Creative Commons Attribution 4.0 International License (http://creativecommons.org/licenses/by/4.0/), which permits unrestricted use, distribution, and reproduction in any medium, provided you give appropriate credit to the original author(s) and the source, provide a link to the Creative Commons license, and indicate if changes were made. The Creative Commons Public Domain Dedication waiver (http://creativecommons.org/publicdomain/zero/1.0/) applies to the data made available in this article, unless otherwise stated. 


\section{Background}

Global cerebral ischemia (GCI) is well known to result in significant neurological and cognitive defects in animals and humans [1]. GCI has multiple causes, including cardiac arrest, asphyxiation, and hypotensive shock. The hippocampal CA1 region is highly vulnerable to damage from GCI, resulting in significant delayed death of hippocampal pyramidal neurons [2, 3]. Currently, there are no effective therapies for preserving cognitive function after global cerebral ischemia. Therapeutic hypothermia has been used clinically, but a meta-analysis failed to find a strong benefit on survival or neurological outcome [4]. Thus, there is a clear need to find new potential therapies for preserving cognitive function after GCI.

In previous studies, we and others showed that activation of G-protein-coupled estrogen receptor (GPER) is strongly neuroprotective in an animal model of GCI [5-7]. GPER, also known as GPR30, is the most recently identified member of the estrogen receptor family. Beginning with studies published in 2000, it was found that GPER binds the potent estrogen, $17 \beta$-estradiol (E2), and helps mediate E2-induced rapid activation of extracellular regulated kinases (ERKs) and cAMP generation [8-10]. GPER is highly localized in the cerebral cortex and hippocampus of the brain, with strong expression also noted in the basal forebrain, thalamus, and dorsal striatum $[5,11]$. Studies to explore the role and functions of GPER in the brain have employed G1, a specific agonist, and either G15 or G36, GPER antagonists [12, 13]. GPER shRNA and antisense oligonucleotide knockdown have also been employed in several studies [5, 14]. Intriguingly, we and others previously reported that GPER activation via G1 administration can rapidly activate PI3K-Akt and MEK-ERK rapid kinase signaling pathways in the hippocampus and exert strong neuroprotection against GCI [5-7]. We also showed that E2 neuroprotection against GCI could be abolished by GPER knockdown in the hippocampal CA1 region [5].

Neuroinflammation can also contribute significantly to neuronal cell death in neurodegenerative disorders and in cardiac arrest, and attenuation of neuroinflammation can be neuroprotective $[15,16]$. An important advance in the neuroinflammation field was the identification of inflammasomes as critical proteins that trigger neuroinflammation [17]. NLRP3 inflammasome is the most studied inflammasome in the CNS. It is a multiprotein complex that mediates activation of caspase- 1 and promotes secretion of pro-inflammatory cytokines, such as interleukin-1 $\beta$ (IL-1 $\beta$ ) and interleukin-18 (IL-18) [18]. After activation and release, the biological actions of IL$1 \beta$ are known to be mediated by the type 1 IL-1 receptor (IL-1R). Intriguingly, an endogenous IL-1 receptor antagonist (IL1RA) has been identified in neurons that can block IL-1 $\beta$ pro-inflammatory actions by competing with IL-1 $\beta$ for IL-1R binding. Thus, IL1RA has been reported to exert potent anti-inflammatory and neuroprotective actions in the brain and other tissues [19-21].

Since neuroinflammation is implicated to play a significant role in neurodegeneration, we hypothesized that GPER neuroprotective effects in GCI could be due, in part, to anti-inflammatory actions of GPER. We thus examined the ability of GPER activation to regulate microglial activation, NLRP3 inflammasome activation, and IL-1 $\beta$ production in the hippocampus after GCI. In addition, we examined whether GPER activation could regulate IL1RA in the hippocampus after GCI and determined whether IL1RA mediates the anti-inflammatory, neuroprotective, anti-apoptotic, and cognitive-enhancing effects of GPER following GCI.

\section{Materials and methods Antibodies and reagents}

The following antibodies were used: NeuN (Millipore Biotechnology, MAB377); GPER (sc-48525); Iba1 (sc32725), GAPDH (sc-32233), NLRP3 (Life science, Lot L27693), NLRP3 (ab4207), ASC (sc-22514-R), CD 11b (Gentex, GTX76060), cleaved caspase 1 (cell signaling, \#4199S), cleaved IL1 $\beta$ (sc-7884), IL1RA (ab124962), and IL1RA blocking peptide (ab200257); and NF-kB-p65 (ab32568), H2A (ab177308), Bcl2 (sc-492), cleaved caspase 3 (D175, 5A1E), CREB (cell signaling, \#9197X), and p-CREB (cell signaling, \#9198S), tubulin (sc-9104). Alexa-conjugated secondary antibodies were from Molecular Probes/Invitrogen (Carlsbad, CA). Polyvinylidene difluoride (PVDF) membranes with pore size of $0.45 \mu \mathrm{m}$ were from Millipore (USA). BCIP (5-bromo-4-chloro-3indolyl-phosphate) and NBT (nitro blue tetrazolium) were from Promega (Madison, WI). TUNEL Kits (LOT \#1639496, Life technologies) were from Active Motif Company. Duolink PLA kits (DUO92105), Duolink PLA Rabbit MINUS (DUO92003), and PLA Goat PLUS (DUO92005) proximity probes were from SigmaAldrich. Unless indicated otherwise, all the other chemicals were from Sigma-Aldrich (St. Louis, MO).

\section{Animal and $\mathrm{GCl}$ model}

Adult female Sprague-Dawley rats (Beijing HFK Bioscience Co., Ltd., 3 months old) were housed in a temperature-controlled $\left(22-24{ }^{\circ} \mathrm{C}\right)$ room with water and food freely available. All procedures used in this study were approved by the Institutional Animal Care and Use Committee of North China University of Science and Technology (Ref. 2016047), and were conducted in accordance with the guidelines of the National Natural Science Foundation of China for animal research. The rats were bilateral ovariectomized (OVX) under isoflurane anesthesia and then randomly allocated to each group. In order to reduce bias in the study, a doubleblind procedure was carried out in which drug- 
treatment animals were administrated by blinding investigators and statistical analysis was blindly performed by the authors. A total of 170 rats were used through the study. Of the total number of rats that underwent global cerebral ischemia (GCI), 10 rats died in ischemia reperfusion, and 14 rats were eliminated from further experiment due to not meeting the established criteria for successful cerebral ischemia. GCI animal model was induced at 1 week after OVX by the four-vessel occlusion $(4-\mathrm{VO})$ as our previous description [22, 23]. Briefly, the rats were anesthetized using chloral hydrate $(350 \mathrm{mg} / \mathrm{kg}$, i.p.), then both vertebral arteries were electrocauterized through the alar foramen of the first cervical vertebra and both common carotid arteries (CAA) were exposed followed by closing the incision with a suture. After 24 $\mathrm{h}$, the animals were lightly anesthetized to re-expose and occlude the CAA for 12 min with aneurysm clips. Rats that lost their righting reflex within $30 \mathrm{~s}$ and whose pupils were dilated with unresponsive to light during ischemia were deemed as successful and selected for the experiments. Resumption of carotid artery blood flow was verified visually by releasing the clips. Rectal temperature was maintained at about $36.5-37.5{ }^{\circ} \mathrm{C}$ during the procedure with an incubator. For sham-operated animals, all rats were performed exactly as for ischemic animals except that the CCA were not clamped.

\section{Administration of drugs}

GPER agonist, G1 (Tocris, Cat. No. 3577, $10 \mu \mathrm{g} /$ day), or/ and GPER antagonist, G36 (Tocris, Cat. No. 4759, $10 \mu \mathrm{g} /$ day) was administrated subcutaneously using minipump $(0.25 \mu \mathrm{l} / \mathrm{h}$, Alzet Model 2004) beginning at the time of OVX surgery. For vehicle-treatment group, the same volume cottonseed oil with 1\% DMSO was administrated at the same time-points as G1 or G36 administration. IL1RA antisense oligodeoxynucleotide (AS, 5' -ACCAGCTCATTGCTGGGTAC-3') or scrambled missense (MS, 5' -CCGCGAAAATCGCTTTAGCA-3') was synthesized by Integrated DNA Technologies, Inc. The last 3 bases on both the $5^{\prime}$ and $3^{\prime}$ were endphosphorothioated to limit ODN degradation. IL1RAAS or MS (10 nmol/day) was dissolved in $0.9 \%$ saline and was continuously injected into the left lateral ventricle (anteroposterior, $0.8 \mathrm{~mm}$; lateral, $1.5 \mathrm{~mm}$; depth, $3.5 \mathrm{~mm}$; from bregma) beginning at 3 days prior to ischemia until the end of the experiments (Alzet minipump $0.5 \mu \mathrm{l} / \mathrm{h}$, model 2002 , or $1 \mu \mathrm{l} / \mathrm{h}$, model 2001) using brain infusion kit (Alzet, Lot no 10331-14).

\section{Histological analysis}

The rats were anesthetized using isoflurane at reperfusion 1 day, 3 days, and 14 days and transcardially perfused with $0.9 \%$ saline followed by $4 \%$ paraformaldehyde in $0.1 \mathrm{M}$ phosphate buffer (PB, $\mathrm{pH} 7.4)$. The brains were removed and postfixed in the same fixative overnight at $4{ }^{\circ} \mathrm{C}$, followed by dehydration in $30 \%$ sucrose in $0.1 \mathrm{M}$ $\mathrm{PB}$ till completely subsidence of the tissues and then cut longitudinally into $25 \mu \mathrm{m}$ sections with a cryostat. Coronal sections were collected through the entire dorsal hippocampus (2.5-4.5 $\mathrm{mm}$ posterior from bregma) to investigate the neuroprotective effect of GPER following GCI by performing in situ apoptosis detection using TUNEL kit as described by the manufacturer with minor modifications, simultaneously carrying out immunofluorescence staining for NeuN. Briefly, the sections were washed using PBS for $30 \mathrm{~min}$, permeabilized with $0.4 \%$ Triton X-100-PBS for $1 \mathrm{~h}$, blocked in $10 \%$ donkey serum for $1 \mathrm{~h}$, and then incubated in anti-NeuN antibody $(1: 800)$ overnight at $4{ }^{\circ} \mathrm{C}$. After rinsing three times over $30 \mathrm{~min}$ with $0.1 \%$ Triton X-100-PBS, the sections were incubated with secondary antibodies (Alexa Fluor 488-nm donkey anti-mouse IgG) at room temperature for $1 \mathrm{~h}$ followed by washing for $5 \times 10 \mathrm{~min}$ in $0.1 \%$ Triton X-100-PBS. The following steps were protected from light. The sections were incubated in TdT reaction buffer A for $10 \mathrm{~min}$ and then in TdT reaction mixture including enzyme solution for $1 \mathrm{~h}$ at $37^{\circ} \mathrm{C}$. After $5 \mathrm{~min}$ of washing with $\mathrm{dH}_{2} \mathrm{O}$, the sections were incubated in Click-iT Plus TUNEL reaction cocktail for $30 \mathrm{~min}$ at $37^{\circ} \mathrm{C}$, washed with $0.1 \%$ Triton 100 -PBS over $20 \mathrm{~min}$, and then mounted on slides covered with water-based mounting medium. Images were captured under laser scanning confocal microscopy (LSCM, Olympus FV1000), and analysis was carried out using Digital imaging software (FV10-ASW 1.5). The number of NeuNor TUNEL-positive CA1 neurons per $250-\mu \mathrm{m}$ length of the medial CA1 pyramidal cell layer was bilaterally counted in five or six sections of different animals. Cell counts from the right and left hippocampus on each of the seven or eight sections were averaged to provide the mean value. A mean $\pm \mathrm{SE}$ was calculated from the data in each group and statistical analysis performed as described below.

\section{Immunofluorescence staining and confocal microscopy}

The coronal sections $(25 \mu \mathrm{m})$ at time-points of reperfusion at 3 days and 14 days were prepared. All steps including washing, permeabilizing, and blocking were the same as described in histological analysis. The sections were incubated in the following primary antibodies overnight at $4{ }^{\circ} \mathrm{C}$ : anti-GPER (1:100), anti-NeuN (1:800), anti-Iba1 (1:1000), anti-CD11b (1:1000), anti-cleavedIL1 $\beta$, anti-NLRP3 (1:100), anti-ASC (1:100), anti-IL1RA (1:200), p-CREB (1:200), or anti-NF-kB-p65 (1:200). After washing for $3 \times 10$ min with $0.1 \%$ Triton $\mathrm{X}-100$ PBS, the sections were incubated with secondary antibodies (Alexa Fluor 568-nm donkey anti-mouse IgG, Alexa Fluor 568-nm donkey anti-rabbit IgG, Alexa Fluor 
488-nm donkey anti-goat IgG, and Alexa Fluor 488-nm donkey anti-mouse IgG) at room temperature for $1 \mathrm{~h}$, followed by a final washing for $5 \times 10 \mathrm{~min}$ in $0.1 \%$ Triton $\mathrm{X}$-100-PBS. If necessary, the nucleus was counterstained using mounting medium with DAPI (Lot ZA0210, Vector Laboratories, Inc. Burlingame, CA 94010). The confocal images were captured on a laser scanning confocal microscope (LSCM, Olympus FV1000) and digital imaging software (FV10-ASW 1.5 Viewer).

\section{Duolink II proximity ligation assay}

The Duolink II in situ proximity ligation assay (PLA) immunoassay was performed as described previously by our group [22, 24]. Briefly, after the same processes of washing, permeabilizing, and blocking as histological analysis, cerebral coronal sections were incubated using anti-NLRP3 (1:100) and anti-ASC (1:100) primary antibodies overnight at $4{ }^{\circ} \mathrm{C}$. The slides were then incubated with Duolink PLA Rabbit MINUS and PLA Goat PLUS proximity probes for $1 \mathrm{~h}$ at $37^{\circ} \mathrm{C}$. Ligation and amplification were carried out using the Duolink in situ detection reagent kit according to the manufacturer's protocol. DAPI was used to counter stain the nucleus. Images were captured in the hippocampal CA1 region under FV1000 LSCM, and red spots represented the interactions between NLRP3 with ASC.

\section{Brain homogenates and subcellular fractionations}

The rats were sacrificed under deep anesthesia at 3 days and 14 days after ischemia. The brains were quickly removed, and the hippocampal CA1 regions of the two sides were micro-dissected on an ice pad. The total cytosolic or nuclear protein fraction isolation was performed as described by our group previously [22]. In brief, the tissues were homogenized in 1-ml ice-cold homogenization buffer consisting of (in mM) 50 HEPES, pH 7.4, $150 \mathrm{NaCl}, 12 \beta$ glycerophosphate, 3 dithiotheitol (DTT), 2 sodium orthovanadate $\left(\mathrm{Na}_{3} \mathrm{VO}_{4}\right), 1$ EGTA, $1 \mathrm{NaF}, 1$ phenylmethylsulfonyl fluoride (PMSF), 1\% Triton X-100, and inhibitors of proteases and enzymes (Thermo Scientific, Rockford, IL150825, USA) with a Teflon-glass homogenizer. The homogenates were centrifuged at $15,000 \mathrm{~g}$ for $30 \mathrm{~min}$ at $4{ }^{\circ} \mathrm{C}$ to get a total fraction in the supernatants. When necessary, cytosol and nuclear fractions were extracted. Briefly, tissues were homogenized in ice-cold buffer A containing (in $\mathrm{mM}$ ) 10 HEPES, pH 7.9, 1 DTT, $1 \mathrm{Na}_{3} \mathrm{VO}_{4}$, and inhibitors of proteases and enzymes, and mixed and then allowed to swell on ice for $10 \mathrm{~min}$. The tubes were vigorously vibrated for $30 \mathrm{~s}$ and centrifuged at $15,000 \mathrm{~g}$ for $30 \mathrm{~min}$ at $4{ }^{\circ} \mathrm{C}$. The supernatants contained the cytoplasm fraction, and the pellets were washed three times with buffer A and re-suspended in buffer B [(in mM) 20 HEPES, pH 7.9, $400 \mathrm{NaCl}, 20 \%$ glycerine, 1 DTT, $1 \mathrm{Na}_{3} \mathrm{VO}_{4}$ ] with inhibitors of proteases and enzymes. After adding NP-40 to $0.6 \%$ of total solution, the tubes were vigorously rocked at $4{ }^{\circ} \mathrm{C}$ for $30 \mathrm{~min}$ on a rotator and centrifuged at $12,000 \mathrm{~g}$ for $15 \mathrm{~min}$ at $4{ }^{\circ} \mathrm{C}$ to get the supernatants, which contained the nuclear fractions, and all samples were stored in liquid nitrogen until use. The protein concentrations were determined by enhanced $\mathrm{BCA}$ Protein Assay Kit with bovine serum albumin (BSA) as standard.

\section{Western blotting analysis}

Protein samples were heated at $100{ }^{\circ} \mathrm{C}$ for 5 min with loading buffer containing $0.125 \mathrm{M}$ Tris- $\mathrm{HCl}(\mathrm{PH} 6.8$ ), $20 \%$ glycerol, $4 \%$ SDS, $10 \%$ mercaptoethanol, and $0.002 \%$ bromphenol blue, then separated by sodium dodecyl sulfate-polyacrylamide gel electrophoresis (SDS-PAGE) using $10 \%$ acrylamide gels $(50 \mu \mathrm{g}$ per lane). Then, the proteins on the gel were transferred into a PVDF membrane using a wet transfer system, followed by blocking for $1 \mathrm{~h}$ in $3 \% \mathrm{BSA}$ and then incubated overnight at $4{ }^{\circ} \mathrm{C}$ with the following primary antibodies: NLRP3 (1:500), ASC (1:200), GPER (1:500), Iba1 (1:1000), CD11b (1: 1000), p65 (1:1000), bcl2 (1:200), cleaved-IL1 $\beta$ (1:200), cleaved caspase 1 (1:200), cleaved caspase 3 (1:1000), IL1RA (1:500), GAPDH (1:1000), CREB (1:1000), pCREB (1:1000), and tubulin (1:500). The membranes were washed using $0.2 \%$ tween-20 in Tris-buffered saline (TBST) for at least $30 \mathrm{~min}$ at room temperature followed by incubation in HRP-conjugated secondary antibodies for $1 \mathrm{~h}$ at room temperature. Bound proteins were visualized using a CCD digital imaging system, and semiquantitative analyses of the bands were performed with the Image 1.49 analysis software. Band densities for the targeted proteins were normalized to loading controls (GADPH or $\beta$-tubulin). Normalized means were then expressed as fold changes of the corresponding value for control (sham operated) animals. A means \pm SE was calculated from the data for graphical presentation and statistical comparison.

\section{Primary neuron culture}

Primary hippocampal neurons were micro surgically isolated from SD rats at embryonic day 18 (E18) as our previous description [25]. Briefly, dissociated cells were plated on glass coverslips in presence of $10 \mu \mathrm{g} / \mathrm{mL}$ polyD-lysine (Sigma)-coated 24-well culture plats at a density of $4.50 \times 10^{5}$ cells $/ \mathrm{mL}$. Neurobasal medium supplemented with 2\% B-27 (GIBCO, ThermoFisher Scientific, Milan Italy), $0.5 \mathrm{mM}$ glutamine, $25 \mu \mathrm{M}$ glutamate, penicillin/streptomycin (100 units/100 $\mu \mathrm{g}$ for $\mathrm{mL}$ ), and an antimycotic agent, Amphotericin B was used for the maintenance of cell cultures.

On day 2 post-plating, half-volume medium was routinely replaced every 3 days, and neuron culture was maintained in a humidified incubator in an atmosphere of $5 \% \mathrm{CO}_{2}$ at $37^{\circ} \mathrm{C}$. After 8 days, cells were submitted to 
the treatments. For G1-treatment, the neurons were incubated with the culture medium including $1 \mu \mathrm{MG} 1$ final concentration for $30 \mathrm{~min}, 3 \mathrm{~h}, 6 \mathrm{~h}, 24 \mathrm{~h}$, and $48 \mathrm{~h}$, respectively. The time-point of $6 \mathrm{~h}$ was selected for pretreatment with G36 $(10 \mu \mathrm{M}) 20$ min prior to stimulation with G1. Cottonseed oil with 0.1\% DMSO final concentration was the control.

\section{Barnes maze test}

Long-term spatial learning and memory was evaluated by the use of the Barnes maze, which is a widely accepted test of hippocampus-dependent spatial reference memory in rodents. The apparatus consisted of a circular platform of $120-\mathrm{cm}$ diameter elevated $1 \mathrm{~m}$ above the floor, with 18 holes around the perimeter and a recessed chamber (black escape box also called target hole, $20 \times$ $15 \times 12 \mathrm{~cm}$ ) located under one of the holes. During the testing, rats learn the spatial location of the target hole. The maze was surrounded by curtains on which there were visual cues to learn the position of the target hole. The maze testing was performed as described previously by our group with minor alterations [26, 27]. Briefly, the test included three parts, pre-training was carried out at 7 days of reperfusion, followed by 3 days (reperfusion 7 days, 8 days, 9 days) latency trial, and 24 h later, a probe trial was performed (reperfusion 10 days). During the maze testing, the room is lit with a bright flood incandescent light (500 W, $1000 \mathrm{~lx}$ ) shining down on the maze center and a buzzer ( $85 \mathrm{~dB}$ ) turning on. For the pre-training test, the rat was placed in the center of the open platform surface in a black colored cylindrical start chamber. After $10 \mathrm{~s}$ elapsed, the chamber was lifted and the rat was pre-trained to enter the escape box by guiding it to the escape box and remaining there for $2 \mathrm{~min}$. Following the pre-training, the latency trial started and was repeated four times each day. At the beginning of each trial, the rat was placed in the same start chamber, and $10 \mathrm{~s}$ after the onset of the light and buzzer, the chamber was lifted and the rat was free to explore the maze. The trial ended when the rat entered the escape box, or after $3 \mathrm{~min}$ exploration, it failed to find the target hole. The light and buzzer were turned off once each trial ended, and the rat was allowed to stay in the chamber for $1 \mathrm{~min}$ for habituation. Trails were recorded by a camera located overhead of the platform, and the escape latency, escape velocity, and the time spend in the target quadrant (quadrant occupancy) were computerized using ANY-maze analyzer software. The platform was cleaned with $70 \%$ ethanol and dried with a blower fan after each trial. The probe trial was carried out on day 10 of reperfusion. In the trial, the escape box was removed and the time spent in the target quadrant where the escape box had been recorded during a 90-s period.

\section{Novel object recognition (NOR)}

The apparatus for the NOR task consisted of an opaque box measuring $50 \mathrm{~cm} \times 50 \mathrm{~cm} \times 40 \mathrm{~cm}$ high. The test includes three stages: habituation training was carried out on day 11 and day 12 after ischemia, object familiarization trial was carried out on day 13 after ischemia, and then $24 \mathrm{~h}$ later, the NOR trial was conducted on day 14 after ischemia. The rats were first acclimated to the chamber for two consecutive days ( $5 \mathrm{~min}$ each day) prior to testing to explore the empty box. For the object familiarization testing, the rat was placed in the empty box for $1 \mathrm{~min}$, and then, it was removed, and two identical objects $(10 \mathrm{~cm}$ width, 10 $\mathrm{cm}$ height) were centrally fixed to the floor of the box situated $10 \mathrm{~cm}$ apart. The rat was then placed back in the box and allowed to explore for $5 \mathrm{~min}$. The rat was repeatedly exposed to the same two identical objects twice a day at 60 min interval. Twenty-four hours later, the rat was returned to the object recognition box containing a copy of the object from the familiarization stage and a novel object that varied in color and size to test a long-term recognition memory. Object exploration was scored when the rat's nose was within $2 \mathrm{~cm}$ of the object. Object exploration was not scored when the rat used the object to rear upward with the nose of the rat facing the ceiling. The time spent exploring each object and the discrimination index percentage (the percentage time spent exploring the new object) was recorded and analyzed using ANY-maze video tracking software as previously mentioned.

\section{Statistical analysis}

Statistical analysis was performed using one-way analysis of variance (ANOVA) or two-way ANOVA followed by Student-Newman-Keuls tests to determine group differences. When only two groups were compared, a Student's $T$ test was used. Statistical significance was accepted at the $95 \%$ confidence level $(P<0.05)$. Data were expressed as means $\pm \mathrm{SE}$.

\section{Results \\ GPER activation decreases $\mathrm{GCl}$-induced neuroinflammation in the hippocampal CA1 region}

In order to determine whether GPER signaling can exert anti-inflammatory effects after GCI, we first examined Iba1 expression and GPER distribution, as well as the effects of GPER agonist G1 or antagonist G36 at reperfusion 14 days after GCI in the hippocampal CA1 region. Ibal was examined as it is a well-known marker of microglia that is upregulated upon activation of microglia due to inflammation. As shown in Fig. 1a-c, doubleimmunofluorescence staining for Iba1 (red) and GPER (green) in the hippocampal CA1 region revealed that ischemia/reperfusion (IR) 14 days induced a robust enhancement of Iba1 immunofluorescence intensity as well as higher co-localization (yellow) of Ibal with GPER 


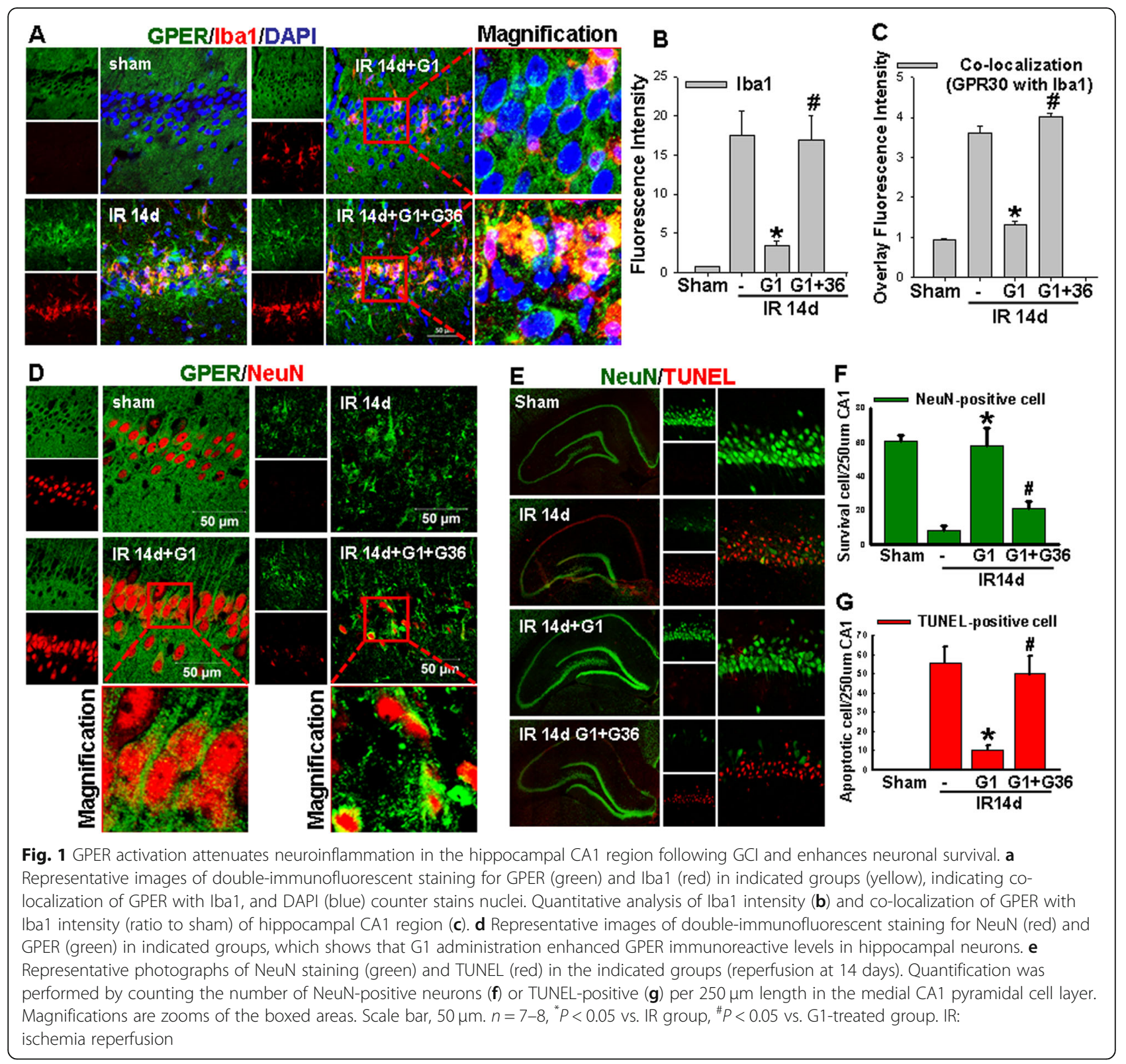

than that in non-ischemic (sham) animals. G1 administration markedly attenuated the Iba1 immunofluorescence intensity and the co-localization of Iba1 with GPER, while G36, a GPER antagonist, reversed the effect of G1 on Iba1 immunofluorescence intensity and colocalization of Iba1 with GPER in the hippocampal CA1 region after 14 days of reperfusion. DAPI (blue) staining was used to visualize the nucleus of cells. Furthermore, double-immunofluorescent staining of GPER (green) with NeuN (a marker of survival neurons, red) indicated that G1-treatment enhanced GPER immunoreactive levels in neurons in the hippocampal CA1 region (Fig. 1d). We next examined the effect of GPER activation upon apoptotic cell death in the hippocampal CA1 region at 14 days of reperfusion after GCI. G1 effect upon apoptosis in the hippocampal CA1 region at IR of 14 days was investigated using NeuN staining (green) and TUNEL analysis (red), and quantification of the results is provided in Fig. 1e-g. As expected, the number of survival neurons (NeuN-positive cells) was significantly decreased by G1 treatment, while the number of apoptotic cells (TUNEL-positive cells) was markedly increased compared to IR group animals, and G36 administration significantly abolished the antiapoptotic effects of G1. Altogether, the results indicate that GPER exerts an anti-inflammatory regulatory effect that may contribute to its neuroprotective effect after GCI. 
NLRP3-ASC inflammasome activation in the hippocampal CA1 region after $\mathrm{GCl}$ is decreased by GPER activation The NLRP3 inflammasome is a major mediator of inflammation that is known to be activated by oxidative stress and inflammatory damage signals, resulting in caspase-1-dependent secretion of the pro-inflammatory cytokine IL-1 $\beta[28,29]$. We hypothesized that GPER signaling may regulate NLRP3 inflammasome activation as one of its anti-inflammatory mechanisms in the brain. To explore our hypothesis, we examined protein expression of the inflammasome components, NLRP3, and ASC in the hippocampal CA1 region at 14 days of reperfusion. Representative photomicrographs for doubleimmunofluorescent staining for NLRP3 (green) and Iba1 (red) are shown in Fig. 2a. The results show that the immunofluorescent intensity of NLRP3 and Iba1 are robustly enhanced and strongly co-localized in the 14-day IR group, as compared to the sham control animals. G1 treatment significantly attenuated the GCI enhancement and co-localization of NLRP3 and Iba1 levels, whereas pretreatment with G36 significantly reversed the G1 effects. Likewise, in Fig. 2b, double-immunofluorescent staining of ASC with CD11b, another marker of microglia, showed a similar pattern, with a robust elevation in protein levels of ASC and CD11b, as well as a very strong co-localization of the two proteins at reperfusion of 14 days, as compared to the sham group animals. G1 treatment significantly attenuated the GCI enhancement and co-localization of ASC and CD11b, whereas pretreatment with G36 significantly reversed the G1 effects. Furthermore, Western blot analysis results for NLPR3 and ASC (Fig. 2c) closely mirrored the findings from immunofluorescent staining of NLRP3 and ASC, as G1 prevented the elevation of NLRP3 and ASC on IR of 14 days, and G36 reversed this effect. It is well established that the recruitment and binding of ASC by NLRP3 is an absolute prerequisite for the activation of the NLRP3 inflammasome. Therefore, we performed an in vivo Duolink II in situ proximity ligation assay using primary antibodies for NLRP3 and ASC to detect the interaction between the two proteins. In Fig. 2d, Duolink puncta (red) in the representative photomicrographs indicates the interaction of NLRP3 with ASC in the hippocampal CA1 region. The results showed that G1 treatment significantly decreased Duolink puncta, as compared with the 14-day IR groups. However, pretreatment with G36 caused Duolink puncta to be increased again, reversing the effect induced by G1 administration. DAPI staining (blue) was also used to visualize the nucleus of cells. As a whole, the findings suggest that GPER decreases protein expression of NLRP3 and ASC and prevents the oligomerization of ASC and NLRP3 necessary for activation of the NLRP3 inflammasome after GCI.
GPER activation inhibits "active" caspase 1 and IL1 $\beta$ expression in the hippocampal CA1 region after $\mathrm{GCI}$ It has been demonstrated previously that the NLRP3ASC complex binds caspase 1, thus forming an active inflammasome complex (NLRP3, ASC, and cleaved caspase-1) that produces IL1 $\beta$. The IL1 $\beta$ precursor is cleaved by "active" caspase 1 (Cle-caspase 1) to form mature IL1 $\beta$ (cleaved IL1 $\beta$, Cle-IL1 $\beta$ ) $[28,30,31]$. Thus, next, we investigated whether GPER activation could suppress activation of the NLRP3 inflammasome complex after GCI. As shown in Fig. 3a-d, Western blot analysis revealed that G1 markedly suppressed protein levels of activated caspase 1 (cle-caspase 1), cle-IL1 $\beta$, and CD11b, as compared with the 14-day IR group, while $\mathrm{G} 36$ reversed the effects in the hippocampal CA1 region. Furthermore, double-immunofluorescent staining for cle-IL1 $\beta$ (green) and CD11b (red) confirmed the above Western blot analysis results and showed that cleIL1 $\beta$ was strongly co-localization with CD11b in the 14day IR and G1 + G36 groups, as compared to the sham or G1 groups in the hippocampal CA1 region (Fig. 3e). These findings suggest that NLRP3 inflammasome activation occurs predominately in activated microglia and is suppressed by GPER activation.

\section{GPER regulates expression of endogenous IL1 $\beta$ receptor antagonist (IL1RA) in the hippocampal CA1 region after} $\mathrm{GCl}$

In addition to suppressing NLRP3 inflammasome activation, we hypothesized that GPER activation might also exert anti-inflammatory effects by regulating expression of IL1RA, an endogenous IL1 $\beta$ receptor antagonist. We thus examined IL1RA protein expression in the hippocampal CA1 region using immunofluorescent staining and Western blot analysis. Representative photomicrographs of double-immunofluorescent staining results for IL1RA (green) and CD11b (red), or IL1RA (green) and NeuN (red) in the hippocampal CA1 region, are shown in Fig. 4a, b. The results reveal that IL1RA immunoreactive levels are strongly decreased in the 14-day IR group as compared to the sham controls. Interestingly, G1 strongly upregulated IL1RA immunoreactive protein levels, as compared to the 14-day IR group, and G36 treatment prevented this effect of G1, while in the 14-day IR group, the IL1RA immunoreactive protein present was found to be co-localized with CD11b in microglia. In contrast, using NeuN as a neuronal marker, we found that G1 strongly upregulated IL1RA immunoreactive protein levels in hippocampal CA1 neurons, and this effect was blocked by G36 (Fig. 4a, b). Western blot results for IL1RA showed that G1 treatment significantly elevated IL1RA protein levels compared with the 14-day IR group, and G36 reversed this effect of G1 (Fig. 4c). The specificity of the IL1RA antibody used in the study was confirmed by pre- 


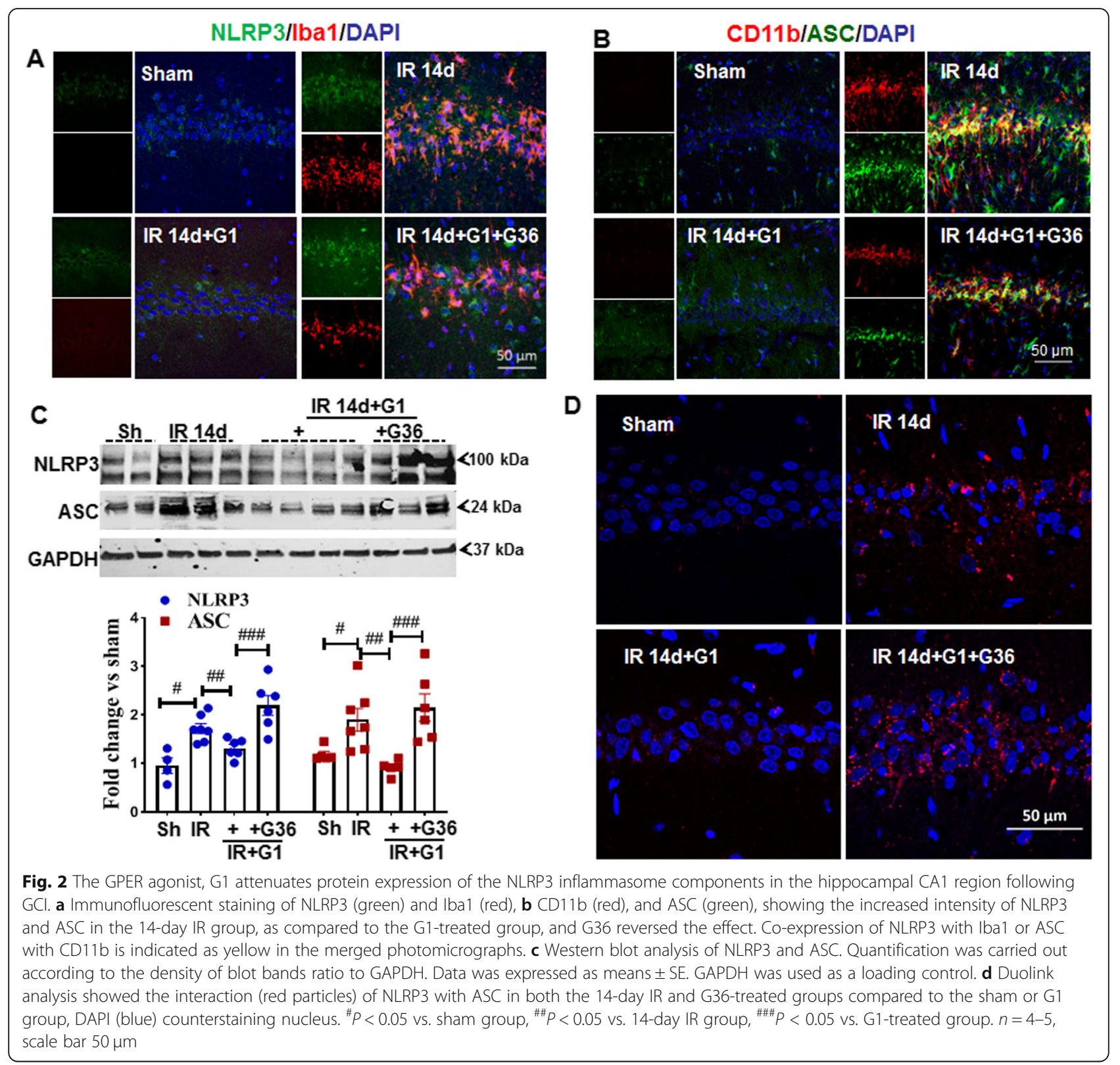

incubation with or without blocking peptide of IL1RA. Western blot for IL1RA using samples from hippocampal CA1 region of sham animals are shown in Fig. 4d. The results demonstrated that in the absence of blocking peptide pretreatment, there was a clear band for IL1RA at around $25 \mathrm{KDa}$ (left line). In contrast, pretreatment with blocking peptide completely blocked the $25 \mathrm{KDa}$ band of IL1RA. In addition, the blocking peptide perfectly abolished staining for IL1RA in the hippocampal CA1 region (green, Fig. 4e), and DAPI (red) staining was used to visualize the nucleus of cells. The results demonstrate the specificity of the IL1RA antibody. They also demonstrate that G1 strongly induces IL1RA immunoreactive protein levels after GCI in neurons in the hippocampal CA1 region, thus potentially "armoring" the neurons against IL1 $\beta$-induced inflammatory damage and cell death. In order to detect whether G1 directly induces IL1RA protein expression, we performed primary hippocampal neuron cultures and treated the neurons with GPER agonist G1. Pretreatment with G1 was for $6 \mathrm{~h}$, and $\mathrm{G} 36(10 \mu \mathrm{M})$ was administered $20 \mathrm{~min}$ prior to stimulation with G1. Cottonseed oil with $0.1 \%$ DMSO final concentration was as the control (C). Western blot analysis showed that there was no significant change in IL1RA protein expression at $30 \mathrm{~min}$ and $3 \mathrm{~h}$ after G1-treatment, whereas G1 markedly enhanced IL1RA expression at $6 \mathrm{~h}$ and $24 \mathrm{~h}$, as compared to the control group (Fig. 4g). G36-administratment reversed the effect induced by G1 at the $6 \mathrm{~h}$ time-point (Fig. 4h). 

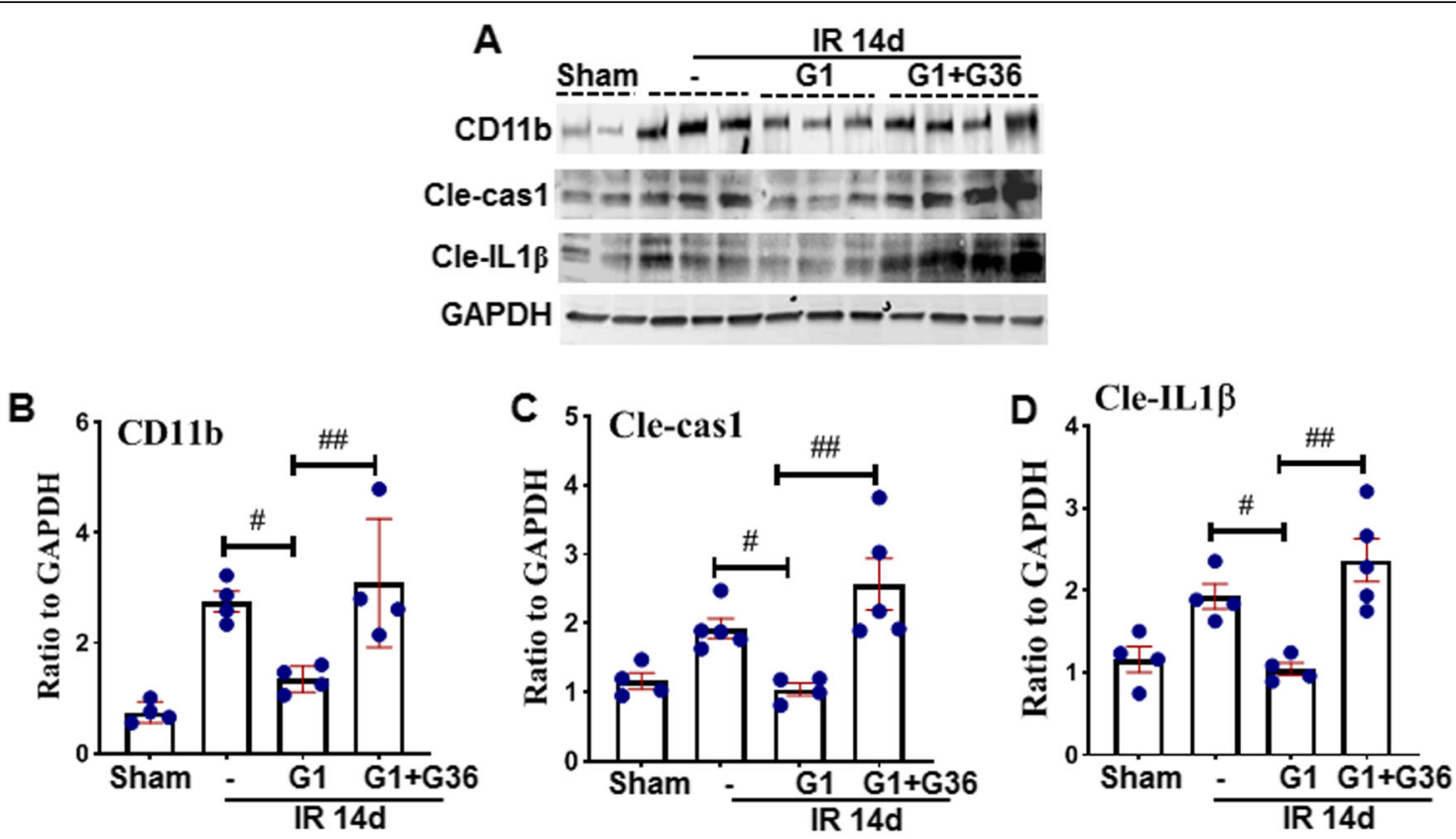

$\mathbf{E}$

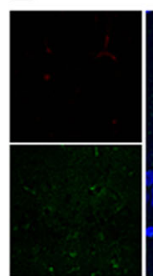

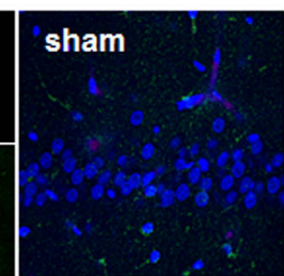

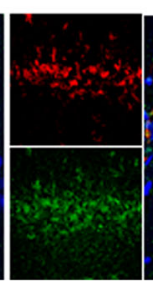

CD11b/Cle-IL1B/DAPI
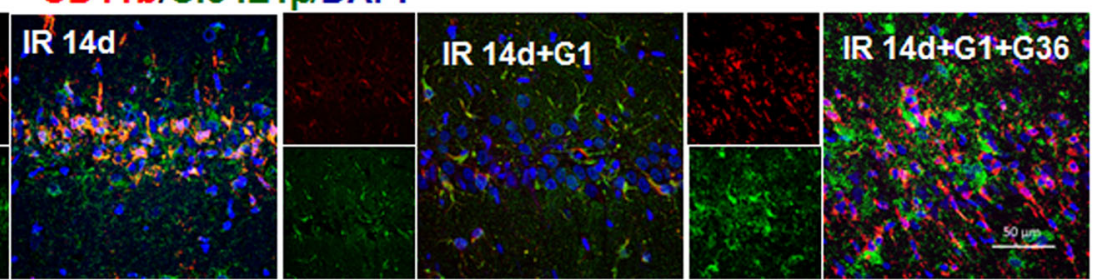

Fig. 3 The effects of the GPER agonist, G1 upon caspase 1 activation and IL1 13 production in the hippocampal CA1 region following GCl. a-d Western blot analysis for CD11b, a marker of activated microglia, Cle-cas1 (cleaved caspase 1) and cleaved IL1 $\beta$. Semi-quantitative analysis was carried out according to the band density of target protein ratio to that of loading control (GAPDH). Data was expressed as mean \pm SE. ${ }^{\#} P<0.05$ vs. 14-day IR group, ${ }^{\# \#} P<0.05$ vs. G1-treated group, e Double-immunofluorescent staining for CD11b (red) and cleaved IL 1b (green), showing a strong increase in CD11b and cleaved IL 1b intensity in the 14-day IR animals compared to the G1-treated group, while G36 reversed the increase. Scale bar $50 \mu \mathrm{m}$, magnification $40 \times n=4-5$

Furthermore, immunofluorescent staining for MAP 2 (neuron marker, red) and IL1RA (green) further confirmed the above results (Fig. 4f).

IL1RA knockdown abolishes the anti-inflammasome effect induced by GPER activation in the hippocampal CA1 region following $\mathrm{GCl}$

We next examined the role of IL1RA in GPER regulation of the NLRP3 inflammasome after GCI. To accomplish this, we centrally administered IL1RA antisense oligonucleotides (IL1RA-AS) into the lateral ventricle to knockdown IL1RA in the hippocampal CA1 region. First, we investigated the effectiveness of IL1RA-AS using G1treated samples. Western blot analysis results for IL1RA are shown in Fig. 5a, which revealed that IL1RA protein expression was markedly decreased by IL1RA-AS, as compared to the G1 + MS control group in the hippocampal CA1 region, thus validating the knockdown effectiveness of the IL1RA-AS. We next determined the effect of IL1RA-AS on the ability of G1 to regulate protein expression of CD11b, NLRP3, and Cle-IL1 $\beta$. As shown in Fig. 5b, c, Western blot analysis indicated that G1-treatment $($ G1 + MS) significantly decreased both NLRP3 and cleIL1 $\beta$ protein expression in the hippocampal CA1 region, as compared with the 3-day IR group, and this effect was profoundly reversed by IL1RA-AS treatment. Furthermore, double-immunofluorescence staining for NLRP3 (or cle-IL1 $\beta$ ) with CD11b showed that similar to 3-day IR group animals, IL1RA-AS administration animals had a markedly enhanced number of CD11b + microglia (red) with fewer and much thicker increase in the size of the cell bodies in the CA1 region, while G1 + MS control animals displayed very few $\mathrm{CD} 11 \mathrm{~b}+$ microglia with a small cell body and elaborated thin processes (Fig. 5d, e). As expected, immunoexpression of NLRP3 was in accordance with the change of CD11b, and co-localization of NLRP3 


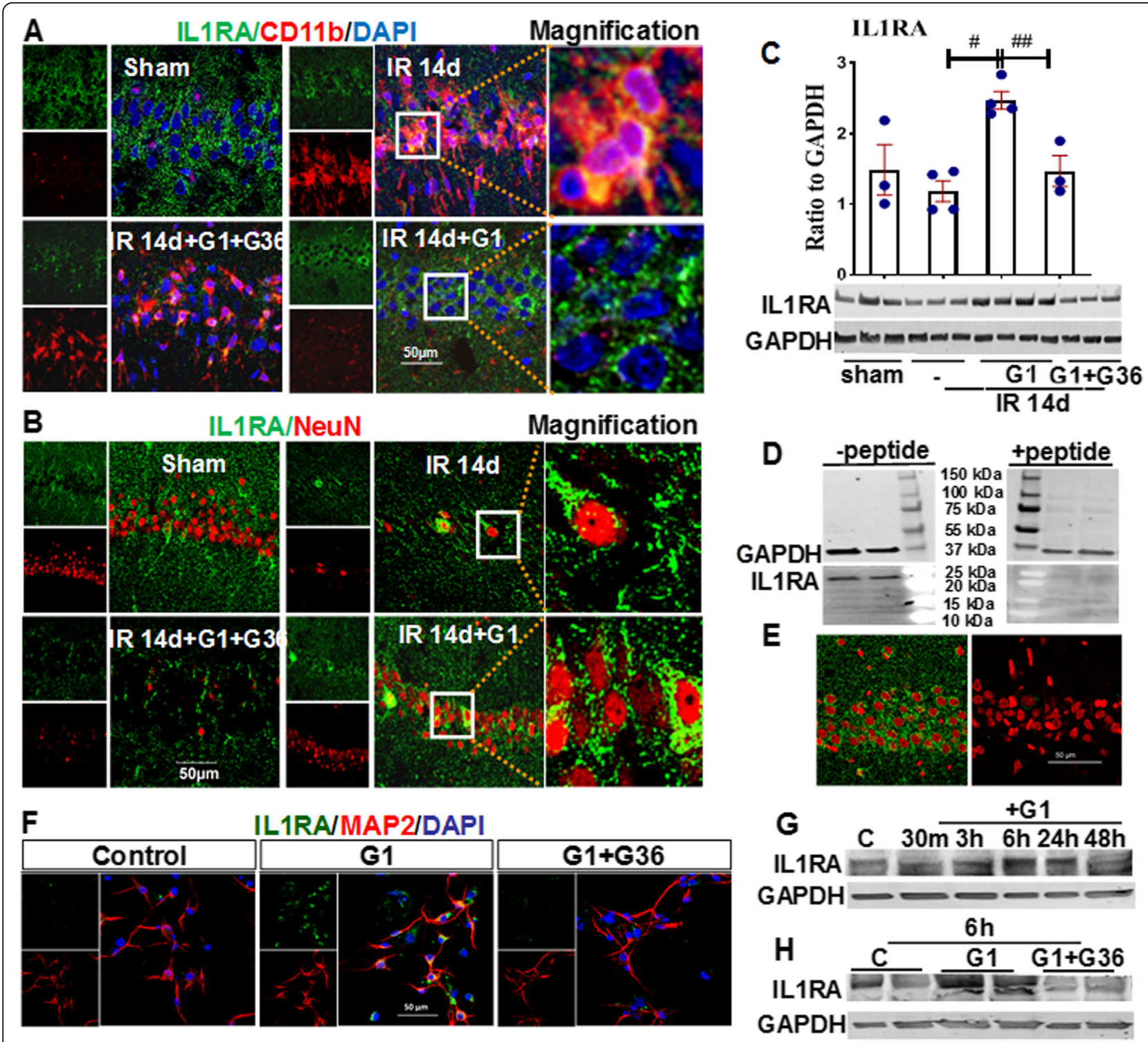

Fig. 4 The effects of the GPER agonist, G1 upon IL1RA immunoreactive protein levels in the hippocampal CA1 region following GCl. a Representative images of double-immunofluorescent staining for a IL1RA (green) and CD11b (red), b IL1RA (green) and NeuN (red). Colocalization of IL1RA immunoreactive protein with the microglia marker, CD11b, and neuronal survival marker, NeuN, is shown in magnification images. DAPI (blue) counter stain for the cell nucleus. c Western blot analysis for IL1RA. ${ }^{\#} P<0.05$ vs. 14-day IR group, ${ }^{\# \#} P<0.05$ vs. G1-treated group. $n=4$ in sham and G1 groups and $n=5$ in IR and G36+G1 groups. d, e The specificity of IL1RA antibody was addressed using Western blot and immunofluorescent staining of IL1RA, and DAPI (red) counter stain for cell nucleus. $\mathbf{f}-\mathbf{h}$ Effect of G1 on IL1RA protein expression in primary hippocampal neurons. Representative photographs of double-immunofluorescence staining for IL1RA (green) and MAP 2 (red) at $6 \mathrm{~h}$ after G1 or G1 + G36-administration, DAPI (blue) counter stain for cell nucleus (f). Western blot analysis showed the IL1RA protein expression in indicated groups $(\mathbf{g}, \mathbf{h}) . \times 40$ magnification, scale $50 \mu \mathrm{m}$

(green) with CD11b (red) (Fig. 5d) or cle-IL1ß (green) with CD11b (red) (Fig. 5e) was seen in the 3-day IR and IL1RA-AS treatment groups rather than in the G1 + MS control and sham groups. As a whole, the results suggest that IL1RA may be an important factor in mediating antiinflammasome activities of GPER following GCI in the hippocampal CA1 region.
G1 inhibits NF-KB-P65 nuclear recruitment in an IL1RAdependent manner

NF- $\mathrm{kB}$ is a major transcription factor that has been implicated as a critical regulator of gene expression in inflammation, particularly in IL-1 production and secretion [32]. Nuclear migration of $\mathrm{P} 65$, a subunit of NF- $\mathrm{BB}$, is pivotal for its activity, and there is evidence that inhibition of NF- 


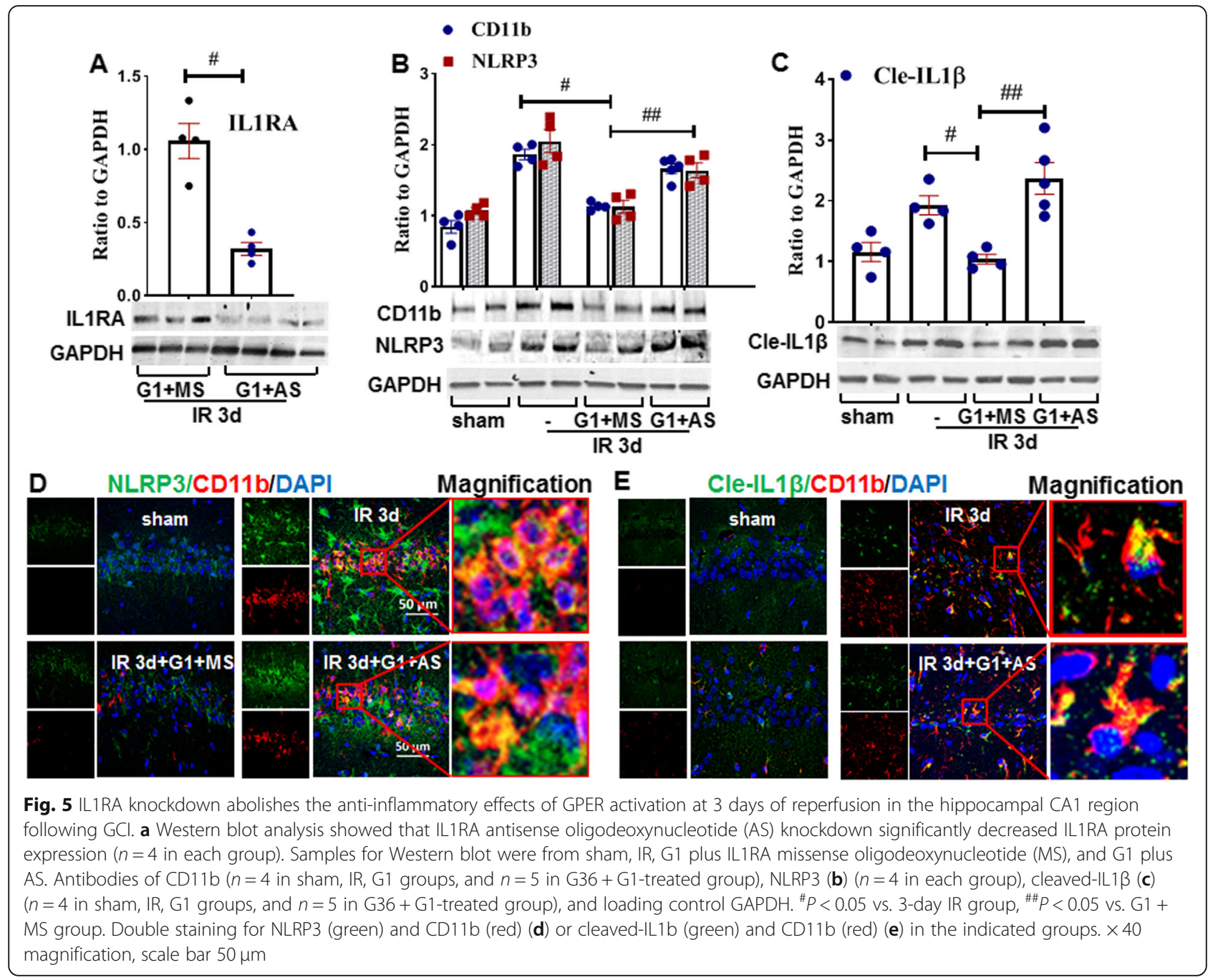

$\kappa B$ inhibits de novo pro-IL1 $\beta[33,34]$. We thus examined the effects of G1 on p65 nucleus translocation at IR of 3 days and further elucidated whether IL1RA is a critical mediator of the process. As shown in Fig. 6a, b, Western blot analysis indicated that $\mathrm{p} 65$ protein levels were significantly decreased in the cytoplasm fraction at the 3-day IR group compared to the sham group, and G1 treatment prevented the decrease. An opposite pattern was observed in the nuclear fraction with p65 protein levels showing a robust enhancement in the 3-day IR group, as compared to the $\mathrm{G} 1+\mathrm{MS}$ control group. Intriguingly, IL1RA-AS profoundly increased p65 protein levels compared to G1 + MS control, although there was no statistical change in the cytoplasm fraction between the two groups. Additionally, Western blot of GAPDH and H2A from both the cytoplasmic and nuclear compartments of sham samples demonstrated the purity of both subcellular fractions (Fig. 6a). Double-immunofluorescent staining for p65 (red) and Iba1 (green) (Fig. 6c, d) showed a robust enhancement of p65 in Iba1+ microglia cells and obviously nuclear expression in the 3-day IR and IL1RA-AS groups, as compared to the sham and G1+MS groups. As a whole, the results indicate that G1 regulates NF- $\mathrm{kB} / \mathrm{p} 65$ protein nuclear translocation in an IL1RA-dependent manner.

\section{Central knockdown of IL1RA protein abolishes the G1- induced anti-apoptotic effect in the hippocampal CA1 region after $\mathrm{GCl}$}

Neuroinflammation is known to occur in parallel with mitochondrial dysfunction and apoptosis. Therefore, we next sought to determine whether G1 regulated mitochondrial apoptosis signaling in the current study, and whether this was dependent upon IL1RA. Western blot analysis showed that $\mathrm{Bcl} 2$, an anti-apoptotic factor was significantly enhanced by G1, as compared to IR of 3 days, and IL1RA-AS knockdown attenuated this effect. Cleaved caspase 3, which is known as an apoptotic executor, displayed an opposite pattern as that of $\mathrm{Bcl} 2$ (Fig. 7) a, b. The transcription factor, CREB, is known to 


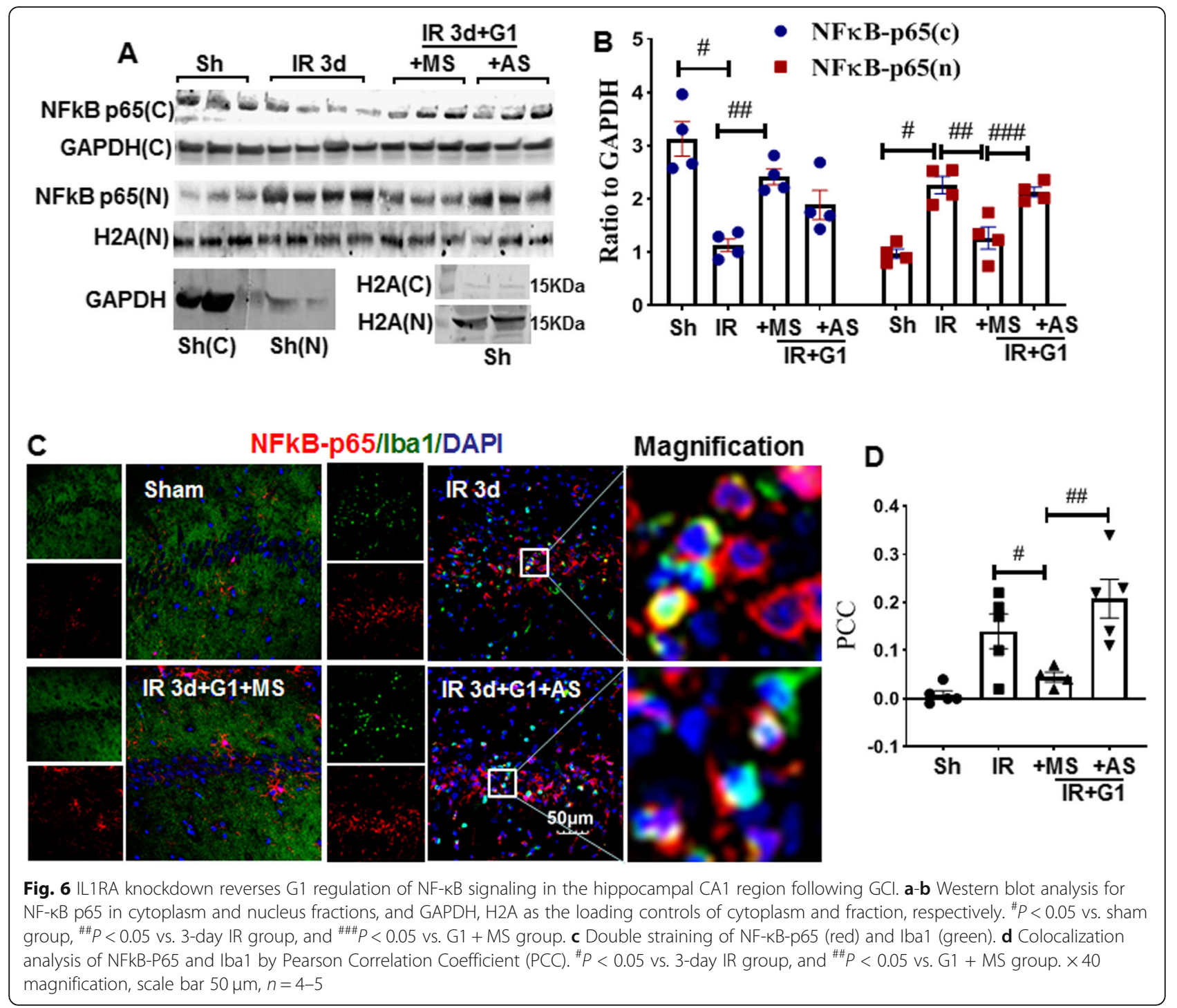

regulate IL1RA expression in the brain [35]. Examination of CREB phosphorylation by Western blot analysis showed that G1-treatment significantly enhanced CREB phosphorylation (activation) compared to the 3-day IR group, while ILIRA knockdown markedly abolished the effect (Fig. 7c). Double-immunofluorescence staining for NeuN and p-CREB mirrored the Western blot results, revealing that $\mathrm{G} 1$ enhanced $\mathrm{p}$-CREB in hippocampal CA1 region neurons and that IL1RA-AS reversed this effect (Fig. 7d). Next, apoptosis detection was carried out using TUNEL analysis, and at the same time, NeuN immunofluorescence staining was performed as a measure of neuronal survival in the hippocampal CA1 region. Figure $7 \mathrm{e}$ shows the representative photographs of NeuN (green) and TUNEL (red) staining in indicated groups, while semi-quantitative analysis of the staining is shown in Fig. 7f, g. The results revealed that $\mathrm{G} 1+\mathrm{MS}$ prevented neuron apoptosis and increased the number of surviving neurons as compared to IR of 3 days, whereas IL1RA-AS significantly abolished these neuroprotective and antiapoptotic effects of G1. Taken together, the findings suggest that GPER activation enhances the defense mechanism in hippocampal neurons after GCI and that this effect requires upregulation of IL1RA.

Central knockdown of IL1RA reverses G1-induced cognitive enhancement following $\mathrm{GCl}$

We next examined whether G1 treatment preserved hippocampal-dependent cognitive function following GCI and whether this effect was dependent upon upregulation of IL1RA. We used the Barnes maze to examine hippocampal-dependent cognitive function at 7-10 days after reperfusion as it is a widely used test to assess spatial reference learning and memory [36, 37]. As shown in Fig. 8a, there was no significant change on day 1 of the latency trial among the groups, while on day 2 


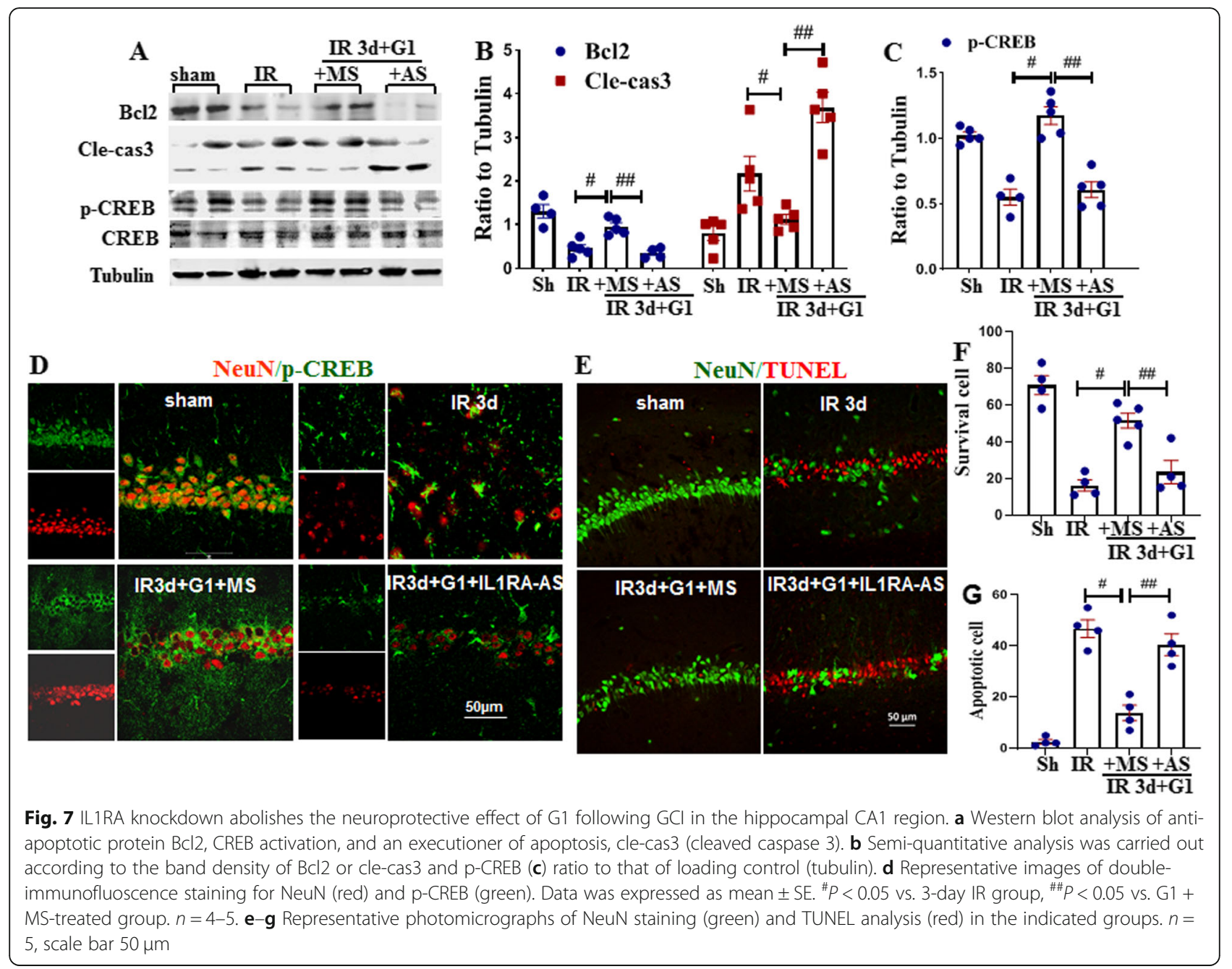

and day 3, reperfusion at 9 days resulted in a significant increase in latency to find the target hole (Fig. 8a), as compared to the sham group, with a significant decrease in the time spent in the escape box quadrant (Fig. 8b). On day 2 of the latency trial, as compared to the IR group, G1 administration decreased the time to find the escape box but this was not statistically significant, while on day 3, G1 significantly decreased the time to find the target hole (Fig. 8a) and increased the time spent in exploring the quadrant where the escape box had been (Fig. 8b). Importantly, administration of either the GPER antagonist G36 or IL1RA-AS reversed the cognitive improvements induced by G1, as evidenced by G36- and IL1RA-AS-treated animals exhibiting a significantly enhanced latency to find the target hole and significantly decreased time spent in the quadrant where the escape box had been, as compared to only G1-treated animals (Fig. 8a, b). Further studies revealed no difference in the exploring speed of the animals (Fig. 8c). Representative tracings indicating sample paths of the rats from the latency trial and probe trial are shown in Fig. 8d, e.
In order to further address the cognitive improvement effects of G1-induced IL1RA signaling, we next performed the novel object recognition test to assess hippocampus-dependent working memory in the rats [38]. Figure 9a shows the protocol of the training day (reperfusion at 13 days) presenting two identical objects in the box, and the testing day (reperfusion at 14 days) presenting one familiar object (Fa-ob) and a novel object (Nov-ob). Figure 9b demonstrates that the time spent by each group of animals in exploring the two objects had no significant differences on training day. However, on testing day, sham and G1-treated animals spent a significantly longer time exploring the novel object than the familiar object. In contrast, the time spent on the familiar and novel objects in IR, G1 + G36treated and G1 + IL1RA-AS-treated animals showed no statistically significant differences (Fig. 9c). Notably, the increased discrimination index of G1 was profoundly decreased by G36 and IL1RA-AS, demonstrating that the effective role of G1 in improving long-term learning and recognition memory following $\mathrm{GCI}$ is mediated by 


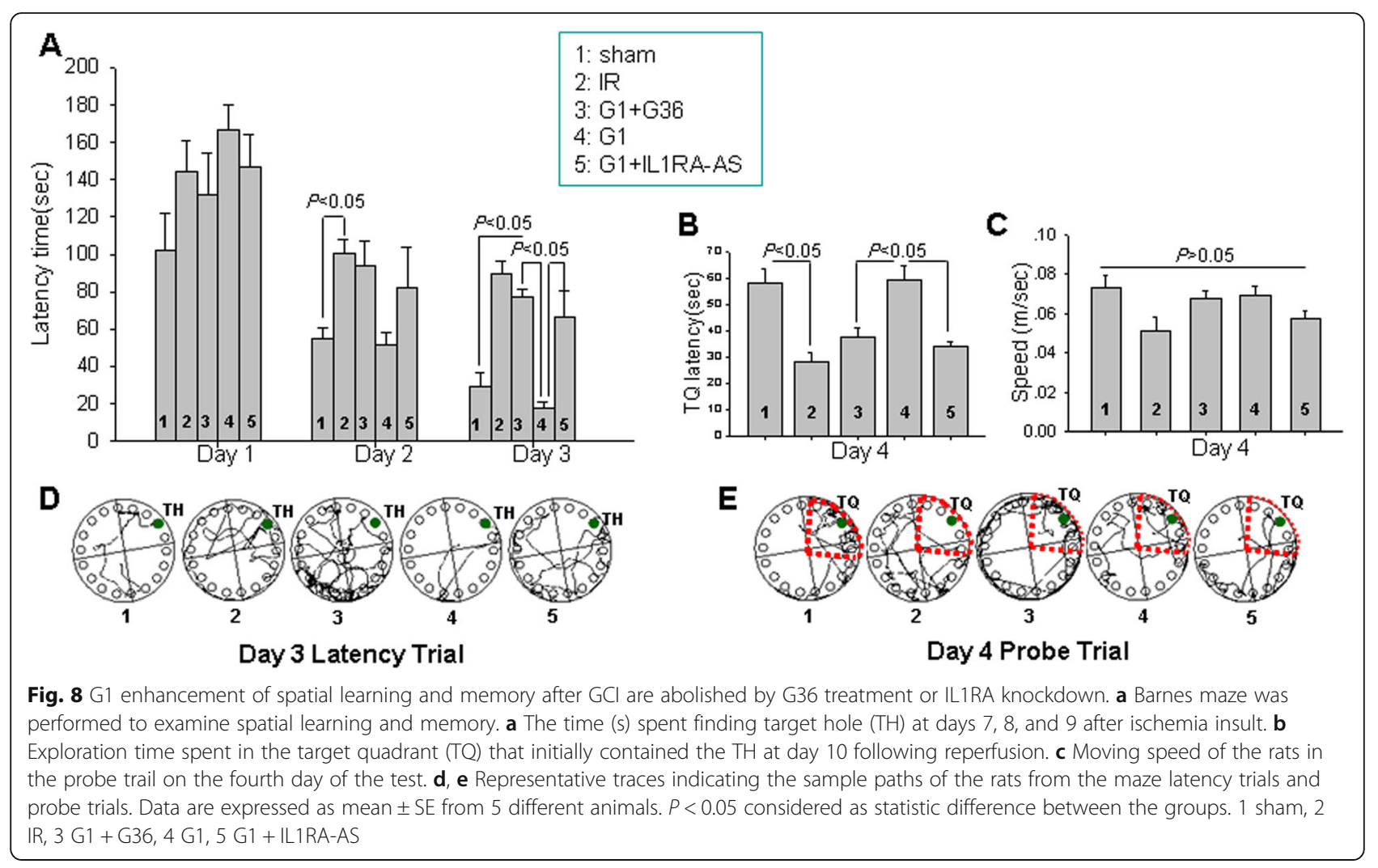

GPER and IL1RA (Fig. 9d). Representative tracings indicating sample paths of the rats from testing day are shown in Fig. 9e.

\section{Discussion}

The current study provides several important findings. First, it demonstrates that GPER activation exerts potent anti-inflammatory effects in the hippocampus after GCI to reduce microglia activation, suppress NLRP3 inflammasome activation, and downstream active IL-1 $\beta$ generation and signaling. Secondly, it demonstrates that GPER activation enhances defense mechanisms in neurons by profoundly upregulating the anti-inflammatory protein and IL1RA in neurons in the hippocampal CA1 region after GCI. Thirdly, it demonstrates that IL1RA is critical for mediating GPER's anti-inflammatory, neuroprotective, anti-apoptotic, and cognitive-preserving effects after GCI.

The results of our study demonstrate a profound antiinflammatory and neuroprotective effect of GPER activation in the hippocampal CA1 region of ovariectomized rats after GCI. Our data suggest that these effects of GPER activation are mediated through effects on both neurons and microglia. Indeed, GPER was shown in this study and other studies to be strongly expressed in both neurons and microglia in the hippocampus and other brain areas [14, 39-42]. To our knowledge, our study is the first to demonstrate that administration of the GPER agonist, G1 to ovariectomized rats, can markedly decrease protein expression and prevent the oligomerization of ASC and NLRP3 in microglia in the hippocampal CA1 region after GCI. These effects of G1 were reversed by the GPER antagonist G36, demonstrating the critical role of GPER for the anti-inflammatory actions of G1 in the hippocampus. The suppression of NLRP3 and ASC expression and oligomerization by G1 after GCI was correlated with significant inhibition of IL-1 $\beta$, a major pro-inflammatory product of the NLRP3 inflammasome. Elevation of IL-1 $\beta$ in the brain has been shown to inhibit synaptic strength and long-term potentiation in vivo [43], and its administration in vitro is neurotoxic [43]. In addition, treatment with an IL-1 $\beta$ neutralizing antibody has been shown to enhance functional cognitive recovery after GCI [44]. In agreement with these results, NLRP3 knockout mice and NLRP3 inhibitor-treated mice have likewise been shown to have significantly reduced infarct size and improved neurological outcome after cerebral ischemia [45-47]. Based on these findings, we propose that GPER inhibition of NLRP3 inflammasome activation and IL- $1 \beta$ in microglia could contribute, in part, to the anti-apoptotic and cognitive-preserving effects of GPER after GCI.

In this study, we only examined the role of GPER in ovariectomized female animals. It is unclear if GPER activation would exert a similar anti-inflammatory role 

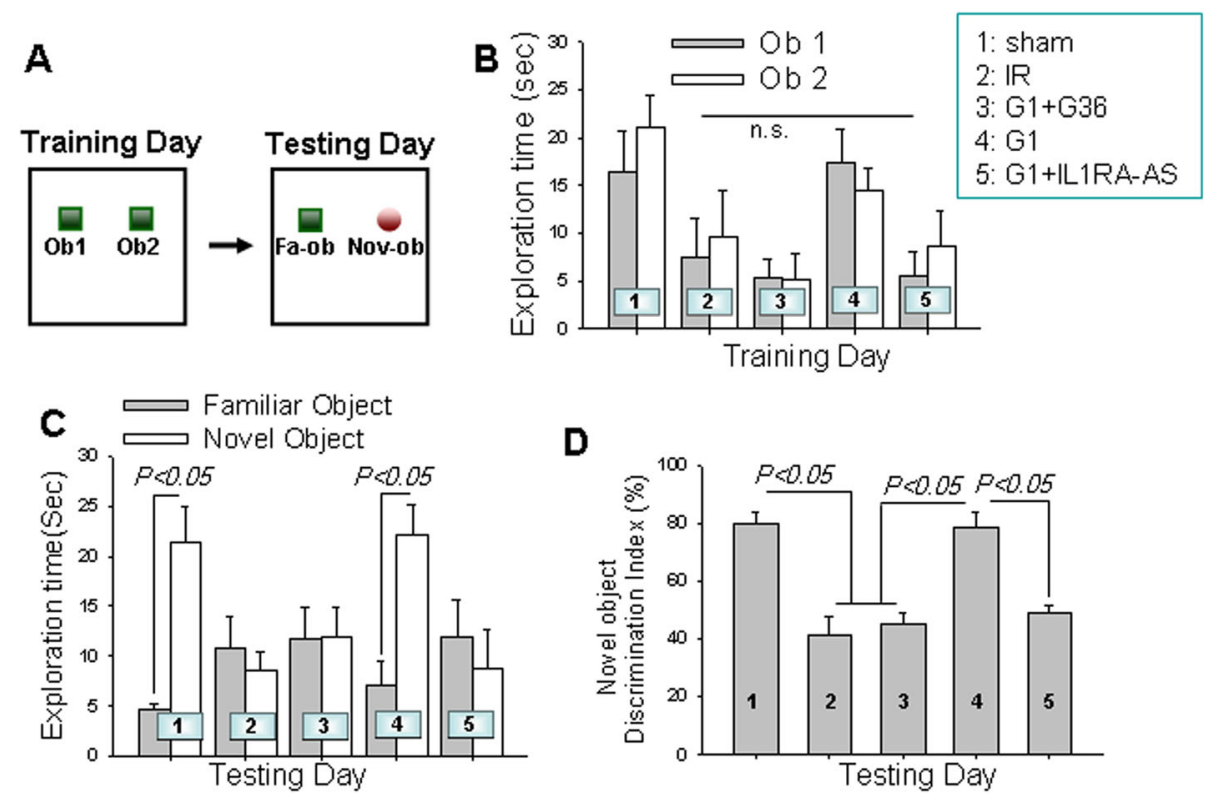

D
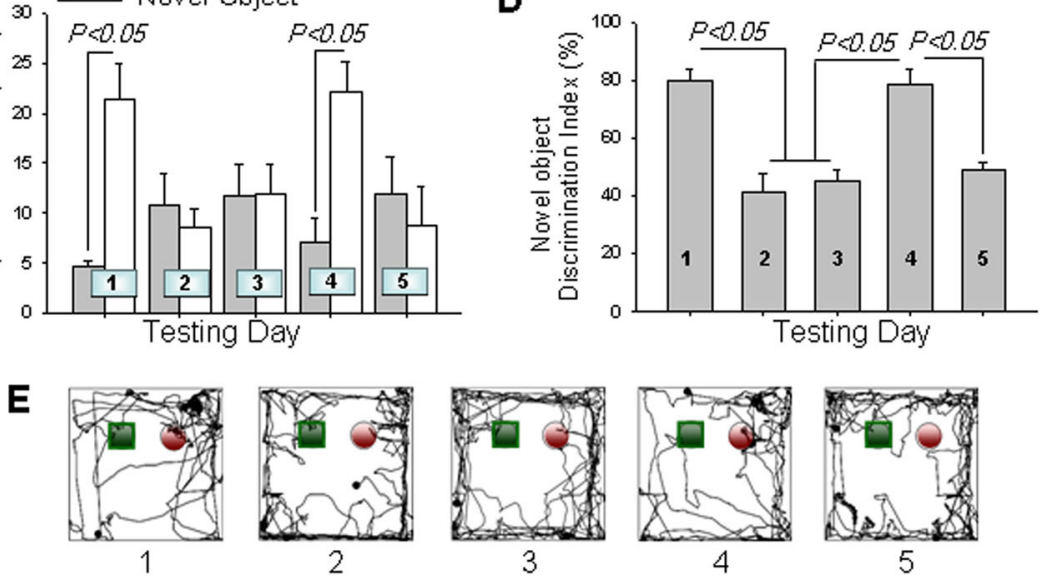

3
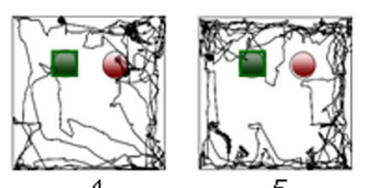

Fig. $9 \mathrm{G} 1$ enhancement of reference memory after $\mathrm{GCl}$ is abolished by G36 treatment or IL1RA knockdown. The novel object recognition (NOR) test was performed following GCl. Five-minute NOR tests at 13 and 14 days after 12-min ischemia were performed to monitor the long-term memory (a). b Time spent exploring the two familiar objectives on the training day. c The time spent exploring each object (familiar object and novel object) and $\mathbf{d}$ the discrimination index percentage (the time spent exploring novel object divided by total time spent). e Representative traces of the indicated groups on the testing day. All the data are expressed as means standard error from 6 to 7 animals in each group. ${ }^{*}<<0.05$ considered as a statistic difference between the groups. n.S., no significant change; Ob, object; Fa-ob, familiar object; Nov-ob, novel object

after GCI in male animals. A review of the scientific literature reveals there is some controversy on whether sex differences exist in GPER actions after cerebral ischemia. For instance, Broughton et al. reported that G1 was only protective in ovariectomized females and not males following focal cerebral ischemia in mice [48]. Moreover, focal cerebral ischemia has been shown to induce GPER in the male mouse brain without altering expression in females [49]. However, another group which used cardiac arrest to induce GCI found that G1 was strongly neuroprotective in male animals [50]. The reasons for the discrepant results are not clear, but it could be due to different ischemia models, different doses, routes of administrations, or pretreatment periods for G1 administration used in the studies. Further work will be needed to more fully clarify potential sex differences in GPER actions in the ischemic brain.

In addition to its anti-inflammatory effects upon microglia, our study identified an additional novel mechanism by which GPER activation could potentially "armor" healthy neurons from inflammatory insults after cerebral ischemia. Using the agonist G1, we showed that GPER activation can markedly elevate expression of IL1RA in hippocampal neurons after GCI. IL1RA has been identified as a natural physiologically occurring negative regulator of inflammation that protects cells from insult $[51,52]$. Thus, G1 induction of IL1RA in hippocampal neurons could serve to protect hippocampal neurons against the damaging effects of IL-1 $\beta$ after GCI. In support of this possibility, our study found that knockdown of IL1RA via central administration of IL1RA antisense oligonucleotides significantly attenuated the neuroprotective, antiapoptotic, and cognitive-enhancing effects of G1 after GCI. This finding suggests that the elevation of IL1RA by G1 is essential for GPER's beneficial neuroprotective and cognitive effects after GCI.

Our finding of G1 enhancing IL1RA in neurons is intriguing as most studies have identified IL1RA as being a microglia-generated protein $[53,54]$, and few have ever studied its expression in neurons. However, treatment with a FDA-approved lipid-lowering drug, gemfibrozil, has been previously reported to directly upregulate IL1RA in mouse cortical neurons in vitro, and this effect strongly protected the neurons from IL-1 $\beta$ insult [35]. 
Furthermore, gemfibrozil induction of IL1RA in neurons was dependent upon enhanced PI3K-Akt signaling and CREB-regulated transcription of IL1RA [35]. We propose that GPER activation may directly regulate IL1RA in neurons via the same mechanism as we previously reported that G1 rapidly elevates PI3K-Akt signaling in hippocampal neurons after GCI [5], and the current study showed that GPER activation also enhanced CREB activation, as indicated by its increased phosphorylation. Consistent with this suggestion, previous studies have confirmed that GPER is expressed in extranuclear locations in hippocampal neurons, such as the plasma membrane, endoplasmic reticulum, and in dendrites $[40,55]$, and thus is well positioned to exert rapid extranuclear signaling. However, we cannot rule out the possibility that G1 could act indirectly in another cell type to induce IL1RA in neurons.

Finally, in addition to blocking the neuroprotective effect of G1, we found that IL1RA knockdown also attenuated the anti-inflammatory effect of G1 after GCI. Specifically, G1 ability to suppress NLRP3, cleaved IL$1 \beta$, and NF- $\mathrm{KB}$ activation in the hippocampal CA1 region after GCI was significantly attenuated by IL1RA knockdown. While the mechanism of this effect remains to be elucidated, we propose that these effects could be due to loss of G1-induced IL1RA "armoring" of neurons, leading to their increased damage and/or demise and release of signals that can activate the NLRP3 inflammasome. In support of this possibility, neuronal damage is known to induce release of danger-associated molecular patterns (DAMPs) such as ATP, ROS, DNA, and HMGB1 that can bind to Toll-like receptors (TLRs) or other receptors on microglia and induce NLRP3 inflammasome activation and active IL-1 $\beta$ production in microglia [56, 57]. Thus, the increased release of DAMPs by damaged neurons could lead to microglia activation and explain why the anti-inflammatory effects of G1 were reversed by IL1RA knockdown.

\section{Conclusion}

The findings of this study shed a new light on the role and underlying mechanisms of GPER control of neuroinflammation after GCI and the potential contribution of these effects to the neuroprotective actions of GPER. As such, our study demonstrates for the first time that GPER's anti-inflammasome, anti-apoptotic, and cognitive-preserving effects in the hippocampal CA1 region after GCI involve upregulation and mediation by the potent anti-inflammatory factor, IL1RA.

\section{Abbreviations}

(GPER/GPR30): G-protein-coupled estrogen receptor; 4-VO: Four-vessel occlusion; ASC: Apoptosis-associated speck-like protein; CAA: Common carotid arteries; CREB: CAMP-response element binding protein; E2: 17 $\beta$ estradiol; GCI: Global cerebral ischemia; IL1RA: Interleukin-1 $\beta$ receptor antagonist; IL-1 $\beta$ : Interleukin-1 $\beta$; NF-KB: Nuclear factor kappa-light-chain-enhancer of activated B cells; NF-KB: Nuclear factor kB; NLRP3: NACHT-, LRR- and PYD-containing protein 3; NOR: Novel object recognition; PLA: Proximity ligation assay

\section{Acknowledgements \\ Not applicable.}

\section{Authors' contributions}

$\mathrm{RM}$ and $\mathrm{DB}$ conceived the study. RM, NB, QZ, WZ, and BL designed and carried out the experiments, and analyzed the data. RM, FY, and DB wrote the manuscript. All authors read and approved the final manuscript.

\section{Funding}

This research was supported by the Natural Science Foundation of China (81671223) and the National Institute of Neurological Disorders and Stroke, U.S. National Institutes of Health (NS050730). The content is solely the responsibility of the authors and does not necessarily represent the official views of the funding bodies.

\section{Availability of data and materials}

All data generated or analyzed during this study are available from the corresponding author on reasonable request.

\section{Ethics approval and consent to participate}

All procedures used in this study were approved by the Institutional Animal Care and Use Committee of North China University of Science (Ref. 2016047) and Technology and were conducted in accordance with the guidelines for animal research of the National Natural Science Foundation of China.

\section{Consent for publication}

Not applicable.

\section{Competing interests}

The authors declare that they have no competing interests.

\section{Author details}

${ }^{1}$ Neurobiology Institute of Medical Research Center, North China University of Science and Technology, Tangshan 063210, Hebei, China. ${ }^{2}$ Department of Neuroscience and Regenerative Medicine, Medical College of Georgia, Augusta University, Augusta, GA 30912, USA. ${ }^{3}$ First Department of Neurology, Hospital Affiliated to North China University of Science and Technology, Tangshan 063000, Hebei, China. ${ }^{4}$ Key Laboratory of Dementia and Cognitive Disorder in Tangshan, North China University of Science and Technology, International Science \& Technology Cooperation Base of Geriatric Medicine of China, 21 Bohai Road, Caofeidian Xincheng, Tangshan 063210, Hebei, China.

Received: 10 December 2019 Accepted: 13 January 2020

Published online: 01 February 2020

References

1. Neumann JT, Cohan CH, Dave KR, Wright CB, Perez-Pinzon MA. Global cerebral ischemia: synaptic and cognitive dysfunction. Curr Drug Targets. 2013;14:20-35.

2. Sekhon MS, Ainslie PN, Griesdale DE. Clinical pathophysiology of hypoxic ischemic brain injury after cardiac arrest: a "two-hit" model. Crit Care. 2017;21:90.

3. Pulsinelli WA, Brierley JB, Plum F. Temporal profile of neuronal damage in a model of transient forebrain ischemia. Ann Neurol. 1982;11:491-8.

4. Kim YM, Yim HW, Jeong SH, Klem ML, Callaway CW. Does therapeutic hypothermia benefit adult cardiac arrest patients presenting with nonshockable initial rhythms?: a systematic review and meta-analysis of randomized and non-randomized studies. Resuscitation. 2012;83:188-96.

5. Tang H, Zhang Q, Yang L, Dong Y, Khan M, Yang F, Brann DW, Wang R. GPR30 mediates estrogen rapid signaling and neuroprotection. Mol Cell Endocrinol. 2014;387:52-8.

6. Lebesgue D, Traub M, De Butte-Smith M, Chen C, Zukin RS, Kelly MJ, Etgen AM. Acute administration of non-classical estrogen receptor agonists attenuates ischemia-induced hippocampal neuron loss in middle-aged female rats. PLoS One. 2010;5:e8642.

7. Lebesgue D, Chevaleyre V, Zukin RS, Etgen AM. Estradiol rescues neurons from global ischemia-induced cell death: multiple cellular pathways of neuroprotection. Steroids. 2009;74:555-61. 
8. Prossnitz ER, Barton M. The G-protein-coupled estrogen receptor GPER in health and disease. Nat Rev Endocrinol. 2011;7:715-26.

9. Prossnitz ER, Barton M. Signaling, physiological functions and clinical relevance of the G protein-coupled estrogen receptor GPER. Prostaglandins Other Lipid Mediat. 2009;89:89-97.

10. Thomas $P$, Pang $Y$, Filardo EJ, Dong J. Identity of an estrogen membrane receptor coupled to a $\mathrm{G}$ protein in human breast cancer cells. Endocrinology. 2005;146:624-32.

11. Alexander A, Irving AJ, Harvey J. Emerging roles for the novel estrogen-sensing receptor GPER1 in the CNS. Neuropharmacology. 2017;113:652-60

12. Dennis MK, Field AS, Burai R, Ramesh C, Petrie WK, Bologa CG, Oprea TI, Yamaguchi Y, Hayashi S, Sklar LA, et al. Identification of a GPER/GPR30 antagonist with improved estrogen receptor counterselectivity. J Steroid Biochem Mol Biol. 2011;127:358-66.

13. Bologa CG, Revankar CM, Young SM, Edwards BS, Arterburn JB, Kiselyov AS, Parker MA, Tkachenko SE, Savchuck NP, Sklar LA, et al. Virtual and biomolecular screening converge on a selective agonist for GPR30. Nat Chem Biol. 2006;2:207-12.

14. Zhao TZ, Ding Q, Hu J, He SM, Shi F, Ma LT. GPER expressed on microglia mediates the anti-inflammatory effect of estradiol in ischemic stroke. Brain Behav. 2016:6:e00449.

15. Singhal G, Jaehne EJ, Corrigan F, Toben C, Baune BT. Inflammasomes in neuroinflammation and changes in brain function: a focused review. Front Neurosci. 2014;8:315.

16. Heneka MT, Kummer MP, Stutz A, Delekate A, Schwartz S, Vieira-Saecker A, Griep A, Axt D, Remus A, Tzeng TC, et al. NLRP3 is activated in Alzheimer's disease and contributes to pathology in APP/PS1 mice. Nature. 2013:493:674-8.

17. Martinon F, Burns K, Tschopp J. The inflammasome: a molecular platform triggering activation of inflammatory caspases and processing of proll-beta. Mol Cell. 2002;10:417-26

18. Leemans JC, Cassel SL, Sutterwala FS. Sensing damage by the NLRP3 inflammasome. Immunol Rev. 2011;243:152-62.

19. Sun M, Brady RD, Wright DK, Kim HA, Zhang SR, Sobey CG, Johnstone MR, O'Brien TJ, Semple BD, McDonald SJ, Shultz SR. Treatment with an interleukin-1 receptor antagonist mitigates neuroinflammation and brain damage after polytrauma. Brain Behav Immun. 2017;66:359-71.

20. Pang $Y$, Tien $L T$, Zhu H, Shen J, Wright CF, Jones TK, Mamoon SA, Bhatt $A$ J, Cai Z, Fan LW. Interleukin-1 receptor antagonist reduces neonatal lipopolysaccharide-induced long-lasting neurobehavioral deficits and dopaminergic neuronal injury in adult rats. Int J Mol Sci. 2015;16:8635-54.

21. Craft JM, Watterson DM, Hirsch E, Van Eldik LJ. Interleukin 1 receptor antagonist knockout mice show enhanced microglial activation and neuronal damage induced by intracerebroventricular infusion of human beta-amyloid. J Neuroinflammation. 2005;2:15.

22. Tu J, Zhang X, Zhu Y, Dai Y, Li N, Yang F, Zhang Q, Brann DW, Wang R. Cellpermeable peptide targeting the Nrf2-Keap1 interaction: a potential novel therapy for global cerebral ischemia. J Neurosci. 2015;35:14727-39.

23. Zhang QG, Raz L, Wang R, Han D, De Sevilla L, Yang F, Vadlamudi RK, Brann DW. Estrogen attenuates ischemic oxidative damage via an estrogen receptor alphamediated inhibition of NADPH oxidase activation. J Neurosci. 2009;29:13823-36.

24. Thakkar R, Wang R, Sareddy G, Wang J, Thiruvaiyaru D, Vadlamudi R, Zhang Q, Brann D. NLRP3 inflammasome activation in the brain after global cerebral ischemia and regulation by 17beta-estradiol. Oxidative Med Cell Longev. 2016:2016:8309031.

25. Wang R, Dong Y, Lu Y, Zhang W, Brann DW, Zhang Q. Photobiomodulation for global cerebral ischemia: targeting mitochondrial dynamics and functions. Mol Neurobiol. 2019;56:1852-69.

26. Lu Y, Dong Y, Tucker D, Wang R, Ahmed ME, Brann D, Zhang Q. Treadmill exercise exerts neuroprotection and regulates microglial polarization and oxidative stress in a streptozotocin-induced rat model of sporadic Alzheimer's disease. J Alzheimers Dis. 2017:56:1469-84.

27. Rosenzweig ES, Barnes CA. Impact of aging on hippocampal function: plasticity, network dynamics, and cognition. Prog Neurobiol. 2003;69:143-79.

28. Abderrazak A, Syrovets T, Couchie D, El Hadri K, Friguet B, Simmet T, Rouis M. NLRP3 inflammasome: from a danger signal sensor to a regulatory node of oxidative stress and inflammatory diseases. Redox Biol. 2015;4:296-307.

29. Abderrazak A, Couchie D, Mahmood DF, Elhage R, Vindis C, Laffargue M, Mateo V, Buchele B, Ayala MR, El Gaafary M, et al. Anti-inflammatory and antiatherogenic effects of the NLRP3 inflammasome inhibitor arglabin in ApoE2.Ki mice fed a high-fat diet. Circulation. 2015;131:1061-70.
30. Yu J, Nagasu H, Murakami T, Hoang H, Broderick L, Hoffman HM, Horng T. Inflammasome activation leads to caspase-1-dependent mitochondrial damage and block of mitophagy. Proc Natl Acad Sci U S A. 2014;111:15514-9.

31. Hoffman HM, Wanderer AA. Inflammasome and IL-1 beta-mediated disorders. Curr Allergy Asthma Rep. 2010;10:229-35.

32. Hiscott J, Marois J, Garoufalis J, D'Addario M, Roulston A, Kwan I, Pepin N, Lacoste J, Nguyen H, Bensi G, et al. Characterization of a functional NFkappa B site in the human interleukin 1 beta promoter: evidence for a positive autoregulatory loop. Mol Cell Biol. 1993;13:6231-40.

33. Klenke C, Widera D, Engelen T, Muller J, Noll T, Niehaus K, Schmitz ML, Kaltschmidt B, Kaltschmidt C. Hsc70 is a novel interactor of NF-kappaB p65 in living hippocampal neurons. PLoS One. 2013;8:e65280.

34. Mattson MP, Camandola S. NF-kappaB in neuronal plasticity and neurodegenerative disorders. J Clin Invest. 2001;107:247-54.

35. Corbett GT, Roy A, Pahan K. Gemfibrozil, a lipid-lowering drug, upregulates IL-1 receptor antagonist in mouse cortical neurons: implications for neuronal self-defense. J Immunol. 2012;189:1002-13.

36. Zheng JF, Patil SS, Chen WQ, An W, He JQ, Hoger H, Lubec G. Hippocampal protein levels related to spatial memory are different in the Barnes maze and in the multiple T-maze. J Proteome Res. 2009;8:4479-86.

37. Patil SS, Sunyer B, Hoger $H$, Lubec G. Evaluation of spatial memory of C57BL/6J and CD1 mice in the Barnes maze, the multiple T-maze and in the Morris water maze. Behav Brain Res. 2009;198:58-68.

38. Antunes M, Biala G. The novel object recognition memory: neurobiology, test procedure, and its modifications. Cogn Process. 2012;13:93-110.

39. Waters EM, Thompson LI, Patel P, Gonzales AD, Ye HZ, Filardo EJ, Clegg DJ, Gorecka J, Akama KT, McEwen BS, Milner TA. G-protein-coupled estrogen receptor 1 is anatomically positioned to modulate synaptic plasticity in the mouse hippocampus. J Neurosci. 2015;35:2384-97.

40. Akama KT, Thompson LI, Milner TA, McEwen BS. Post-synaptic density-95 (PSD-95) binding capacity of G-protein-coupled receptor 30 (GPR30), an estrogen receptor that can be identified in hippocampal dendritic spines. J Biol Chem. 2013;288:6438-50.

41. Matsuda K, Sakamoto H, Mori H, Hosokawa K, Kawamura A, Itose M, Nishi M, Prossnitz ER, Kawata M. Expression and intracellular distribution of the G proteincoupled receptor 30 in rat hippocampal formation. Neurosci Lett. 2008:441:94-9.

42. Brailoiu E, Dun SL, Brailoiu GC, Mizuo K, Sklar LA, Oprea TI, Prossnitz ER, Dun NJ. Distribution and characterization of estrogen receptor $\mathrm{G}$ protein-coupled receptor 30 in the rat central nervous system. J Endocrinol. 2007;193:311-21.

43. Bellinger FP, Madamba S, Siggins GR. Interleukin 1 beta inhibits synaptic strength and long-term potentiation in the rat CA1 hippocampus. Brain Res. 1993;628:227-34.

44. Zhao B, Zou CJ, Zhou P. Delayed administration IL-1 beta neutralizing antibody improves cognitive function after transient global ischemia in rats. Behav Brain Res. 2016;303:53-60.

45. Eloise Lemarchand, Jack Barrington, Alistair Chenery, Michael Haley, Graham Coutts, Judith E. Allen, Stuart M. Allan, David Brough, (2019) Extent of Ischemic Brain Injury After Thrombotic Stroke Is Independent of the NLRP3 (NACHT, LRR and PYD Domains-Containing Protein 3) Inflammasome. Stroke 50 (5):1232-1239

46. Ismael S, Zhao L, Nasoohi S, Ishrat T. Inhibition of the NLRP3-inflammasome as a potential approach for neuroprotection after stroke. Sci Rep. 2018:8:5971.

47. Yang F, Wang Z, Wei X, Han H, Meng X, Zhang Y, Shi W, Li F, Xin T, Pang Q, Yi F. NLRP3 deficiency ameliorates neurovascular damage in experimental ischemic stroke. J Cereb Blood Flow Metab. 2014;34:660-7.

48. Broughton BR, Brait VH, Kim HA, Lee S, Chu HX, Gardiner-Mann CV, Guida E, Evans MA, Miller AA, Arumugam TV, et al. Sex-dependent effects of G protein-coupled estrogen receptor activity on outcome after ischemic stroke. Stroke. 2014;45:835-41.

49. Broughton BR, Brait VH, Guida E, Lee S, Arumugam TV, Gardiner-Mann CV, Miller AA, Tang SC, Drummond GR, Sobey CG. Stroke increases g proteincoupled estrogen receptor expression in the brain of male but not female mice. Neurosignals. 2013;21:229-39.

50. Kosaka Y, Quillinan N, Bond C, Traystman R, Hurn P, Herson P. GPER1/GPR30 activation improves neuronal survival following global cerebral ischemia induced by cardiac arrest in mice. Transl Stroke Res. 2012;3:500-7.

51. Vogt C, Hailer NP, Ghadban C, Korf HW, Dehghani F. Successful inhibition of excitotoxic neuronal damage and microglial activation after delayed application of interleukin-1 receptor antagonist. J Neurosci Res. 2008;86:3314-21.

52. Arend WP, Malyak M, Guthridge CJ, Gabay C. Interleukin-1 receptor antagonist: role in biology. Annu Rev Immunol. 1998;16:27-55. 
53. Pousset F, Palin K, Verrier D, Bristow A, Dantzer R, Parnet P, Lestage J. Production of interleukin-1 receptor antagonist isoforms by microglia in mixed rat glial cells stimulated by lipopolysaccharide. Eur Cytokine Netw. 2000;11:682-9.

54. Eriksson C, Nobel S, Winblad B, Schultzberg M. Expression of interleukin 1 alpha and beta, and interleukin 1 receptor antagonist mRNA in the rat central nervous system after peripheral administration of lipopolysaccharides. Cytokine. 2000;12:423-31.

55. Funakoshi T, Yanai A, Shinoda K, Kawano MM, Mizukami Y. G proteincoupled receptor 30 is an estrogen receptor in the plasma membrane. Biochem Biophys Res Commun. 2006;346:904-10.

56. Zhou K, Shi L, Wang Y, Chen S, Zhang J. Recent advances of the NLRP3 inflammasome in central nervous system disorders. J Immunol Res. 2016; 2016:9238290

57. Fleshner M, Frank M, Maier SF. Danger signals and inflammasomes: stress-evoked sterile inflammation in mood disorders. Neuropsychopharmacology. 2017;42:36-45.

\section{Publisher's Note}

Springer Nature remains neutral with regard to jurisdictional claims in published maps and institutional affiliations.

Ready to submit your research? Choose BMC and benefit from:

- fast, convenient online submission

- thorough peer review by experienced researchers in your field

- rapid publication on acceptance

- support for research data, including large and complex data types

- gold Open Access which fosters wider collaboration and increased citations

- maximum visibility for your research: over $100 \mathrm{M}$ website views per year

At BMC, research is always in progress.

Learn more biomedcentral.com/submissions 